\title{
Computing Equilibrium Prices for a Capital Asset Pricing Model with Heterogeneous Beliefs and Margin-Requirement Constraints
}

\author{
Jun Tong* \\ School of Management, Fudan University, Shanghai 200433 \\ Jiaqiao $\mathrm{Hu}^{\dagger}$ \\ Department of Applied Mathematics and Statistics, Stony Brook University, New York 11794 \\ Jianqiang $\mathrm{Hu}^{\ddagger}$ \\ School of Management, Fudan University, Shanghai 200433
}

\begin{abstract}
The mean-variance capital asset pricing model (CAPM) is a useful mathematical tool for studying a variety of financial problems. In contrast to existing work in the literature, which has primarily focused on deriving analytical solutions under restrictive assumptions, we propose a numerical algorithm for efficiently computing the set of equilibrium prices of a CAPM model with heterogeneous investors and arbitrary margin requirements. We present the mathematical formulation of the CAPM model, derive structural properties of the portfolio selection and excess demand functions, and establish the asymptotic convergence of the proposed algorithm under mild conditions. To illustrate the utility of the algorithm, we perform sensitivity analysis on a simple example to study the impact of marginal requirements and interest rates on the resulting equilibrium prices. Numerical studies are also carried out to compare the performance of the algorithm with that of two other popular methods, namely, the fixed point method and the brand-and-bound algorithm.
\end{abstract}

Key words : (B)Finance; Equilibrium price; Margin requirement; Tâtonnement.

*juntong11@fudan.edu.cn

${ }^{\dagger}$ jqhu@ams.sunysb.edu

${ }^{\ddagger}$ hujq@fudan.edu.cn

(C) 2016. This manuscript version is made available under the Elsevier user license http://www.elsevier.com/open-access/userlicense/1.0/ 


\section{Introduction}

The mean-variance capital asset pricing model (CAPM) is a valuable tool in finance. Although its drawbacks and limitations have been evidenced in empirical studies, CAPM remains popular both in theory and practice due to its simplicity and utility in analyzing a variety of financial problems. The shortcomings of the classical CAPM are mostly related to the requirements that all investors have homogeneous expectations and the underlying market is completely efficient - two idealized assumptions that are unlikely to be met in realistic financial markets. Lintner (1969) was the first to investigate the impact of heterogeneity of investors on CAPM by keeping all other assumptions unchanged. Since then, many studies proceeded along the same line; e.g., Levy (1978); Levy et al. (2006); Fama and French (2007); Chiarella et al. (2013); Shi (2016). One of the major factors that may lead to an inefficient market results from short sale restrictions imposed by the government aiming at stabilizing the volatility of asset prices. In particular, since the outbreak of the financial crisis started in 2007, the market has been seeking to restrict short sales to a greater extent by charging margin requirements, and lenders such as brokage firms usually collect a certain amount of capital from borrowers to prevent themselves from potential losses caused by adverse market movements. This has led to studies focusing on using leverage-constrained models to examine the effect of margin requirements on equilibrium asset prices and trading volumes. For example, Lam et al. (2010) propose a margin scheme on when to change the margin requirement and to what level it should be adjusted. Gârleanu and Pedersen (2011) show that margin requirements may cause securities with identical cash flows to be traded at different prices, leading to price gaps and thus violating the law of one price. Anufriev and Tuinstra (2013) conclude that margin requirements will not change the stability of the market equilibrium; however, when some assets are overvalued, the costs of margin requirements may result in increased mispricing and price volatility. More recently, Rytchkov (2014) considers endogenous time-varying margin requirements and analyzes how these requirements affect the capital market equilibrium. Ma et al. (2015) demonstrate that the well-known "Lintnerian invariance" of short selling, i.e., short selling has no impact on asset prices and trading volumes, may no longer hold when there are heterogeneous margin rules.

Existing studies on pricing and trading effect of margin rules typically require obtaining an optimal solution to the CAPM model or its variants. This is usually done analytically by differentiating each individual's portfolio selection problem and then deriving explicit expressions for the equilibrium prices based on market clearing conditions, see, e.g., Lintner (1969), Levy (1978), 
Chiarella et al. (2013), and He and Shi (2007). However, in the presence of additional margin requirements, the budget constraints for the portfolio selection problems become nonlinear (e.g., Gârleanu and Pedersen (2011)). Although in principle, the resulting problems can still be solved by using the KKT conditions, checking these conditions requires enumerating all possible scenarios when inequality constraints become active, which is only analytically tractable in very simple cases when the size of the problem is small (e.g., Jarrow (1980)). For richer and larger models, finding the equilibrium prices will generally need to be carried out using numerical methods.

There is an abundant body of literature on computational approaches for solving general economic equilibrium problems. Most of these methods rely on optimization theory, including the use of mathematical programming with equilibrium constraints (Luo et al. (1996)), the variational inequality approach (Dafermos (1990) and Jofré et al. (2007)), and algorithms specifically developed for models with linear utility functions (e.g., Garg and Kapoor (2006), Ghiyasvand and Orlin (2012)). The basic idea is to formulate an equilibrium model as a complementarity optimization problem so that standard optimization tools such as gradient descent and branch-and-bound can be applied. The fixed point method, pioneered by Scarf and Hansen (1973), is another major class of iterative algorithms for approximating economic equilibria; see also Eaves (1972), Eaves and Schmedders (1999). In contrast to mathematical programming, a salient feature of fixed point algorithms is that they are often globally convergent regardless of the choice of initial solutions. Although some of these approaches can also be applied to determine the equilibrium prices of CAPM models, it is well known that gradient-based methods will in general lead to locally optimal solutions, whereas popular approaches for problems with complementarity constraints, such as branch-and-bound, may have a worst case complexity that is as high as that of exhaustive search. On the other hand, the fixed point method often involves a discretization of the underlying problem, which may lead to computational difficulties, either resulting in a solution space that is too large or in a solution that is not accurate enough.

In this paper, we propose a derivative-free recursive algorithm for computing the equilibrium price of a CAPM model with heterogeneous investors and arbitrary margin requirements. In particular, we focus on the model proposed in Ma et al. (2015), which falls into the general mean-variance framework of Markowitz (1952). The model has essentially the same setup as that of the early work by Jarrow (1980) except that it allows for additional heterogeneous margin rules for short selling. In addition, it is also consistent with the dynamic models proposed in Gârleanu and Pedersen (2011), Anufriev and Tuinstra (2013), and Rytchkov (2014) when restricted to single periods. Thus, the 
primary reason for considering such a model is that it is typical of a number of alternatives formally investigated in the literature, so that solutions obtained and new insights gained from the model could be easily compared and contrasted with existing findings in the field.

The idea underlying our proposed algorithm is based on the intuition that an excess of demand over supply of a certain asset will lead to an increase in its price, whereas an excess supply would tend to lower the price. Therefore, at each iteration of the recursive procedure, the price of each tradable asset is incremented by a small amount proportional to the difference between the market demand and supply of the same asset. The iteration process continues until little or no further improvement can be made, in which case an approximation of the equilibrium price is obtained. The algorithm has a similar flavor as the tâtonnement method proposed by Walras (1954), which has been widely applied to investigate equilibrium stability in economics. However, the convergence of the tâtonnement process assumes that the market satisfies the gross substitutes property (e.g., Arrow and Hurwicz (1960)), a condition that does not hold in our case due to the intricate correlations among risky assets. We justify the asymptotic validity of the algorithm under mild regularity conditions. In particular, our main theoretical contributions are the establishment of the uniform continuity of the optimal portfolio selection function for each individual investor and the (local) monotonicity of the market excess demand function. The latter ensures demands to move in the opposite direction of prices in the vicinity of an equilibrium point, which in turn implies that the sequence of price vectors generated by the algorithm will converge to the set of equilibrium prices.

To illustrate the application of the proposed algorithm, we use it to conduct an empirical sensitivity analysis on a simple example to study the impact of margin requirements and interest rates on the resulting equilibrium prices. The performance of the algorithm is then further tested on a set of randomly generated problem instances and compared with that of two other methods: the fixed point algorithm of Laan and Talman (1979) and the well-known branch-and-bound algorithm. Numerical results indicate that our algorithm scales well with the problem size and is capable of handling relatively large problem instances involving hundreds of investors and tradable assets within a practical amount of time.

The rest of the paper is structured as follows. In Section 2, we describe the CAPM model with heterogeneous margins and list the assumptions imposed on the model. In Section 3, we give a detailed description of the proposed algorithm. In Section 4, we analyze its asymptotic convergence behavior. Computational results are provided in Section 5 to illustrate the empirical performance of the algorithm. Finally we conclude the paper in Section 6 . 


\section{A CAPM Model with Heterogeneous Margin Requirements}

Suppose there are $K$ investors trading in an economy consisting of $J+1$ tradable securities indexed by $j \in\{0,1, \cdots, J\}$, where $j=0$ represents a riskless asset with risk-free interest rate $r$. We consider a single-period $(t=0,1)$ model. Let $p_{j}$ be the price of asset $j$ at $t=0$ and denote by the random variable $X_{j}$ the price of asset $j$ at $t=1$. At the beginning of period $t=0$, each investor $k$ is endowed with a portfolio that contains $n_{j}^{k} \geq 0$ shares of asset $j, j=0, \cdots, J$. Thus, the aggregate market endowment for security $j$ is $\sum_{k=1}^{K} n_{j}^{k}$ and the initial aggregate wealth of investor $k$ is given by $W_{0}^{k}=\sum_{j=0}^{J} n_{j}^{k} p_{j}$. We assume that there is no restriction on borrowing and lending of the risk-free asset and there is no transaction cost on any trading. However, short selling risky asset $j$ by investor $k$ will incur a margin requirement of $100 \times m_{j}^{k} \%$ of the price of security $j$.

At $t=0$, investor $k$ can rebalance his/her portfolio through operations such as shorting and longing on securities under the initial budget constraint to maximize his/her preference at $t=1$. Let $\phi_{j}^{k}$ be the position of asset $j$ held by investor $k$ after rebalancing. The budget constraint of investor $k$ can be written as follows:

$$
W_{0}^{k}=\sum_{j=1}^{J}\left[p_{j} \phi_{j}^{k-} m_{j}^{k}+p_{j} \phi_{j}^{k+}\right]+\phi_{0}^{k},
$$

where $\phi_{j}^{k-}=\max \left\{-\phi_{j}^{k}, 0\right\}, \phi_{j}^{k+}=\max \left\{\phi_{j}^{k}, 0\right\}$. In (1), the term $p_{j} \phi_{j}^{k-} m_{j}^{k}$ represents the margin requirements incurred when short selling asset $j$ and $p_{j} \phi_{j}^{k+}$ is the cost of buying $\phi_{j}^{k+}$ shares of asset $j$. Note that we have normalized $p_{0}$ to 1 for simplicity. It can be seen that the wealth of investor $k$ at the end of holding period $t=1$ becomes

$$
W_{1}^{k}=W_{0}^{k}+r \phi_{0}^{k}+\sum_{j=1}^{J}\left(X_{j}-p_{j}\right) \phi_{j}^{k}
$$

where we have assumed that the proceeds of the short sale are kept with the brokerage firm and cannot be liquidated before the short position is closed at the end of the period. A zero rebate rate is assumed here. We consider the setting of heterogenous investors and use the mean-variance utility function to measure the preference of an investor over the entire distribution of returns, i.e.,

$$
U^{k}\left(W_{1}^{k}\right)=\mathbb{E}^{k}\left[W_{1}^{k}\right]-\frac{\alpha^{k}}{2} \operatorname{Var}^{k}\left(W_{1}^{k}\right)
$$

where $\alpha^{k}$ is a positive constant representing the degree of risk aversion, $\mathbb{E}^{k}[\cdot]$ and $\operatorname{Var}^{k}(\cdot)$ are the 
expectation and variance operators, which may differ across investors. Thus, by substituting (2) into (3), the optimal portfolio selection problem of investor $k$ can be stated as follows:

$$
\begin{aligned}
\left(I^{k}\right): & \max _{\phi_{j}^{k}, j=0, \cdots, J} \\
\text { subject to } & \sum_{j=1}^{J} \phi_{j}^{k} \mu_{j}^{k}+\sum_{j=1}^{J}\left(m_{j}^{k} \phi_{j}^{k-}+\phi_{j}^{k}-\phi_{j}^{k}\right) p_{j}+r \phi_{0}^{k}+n_{0}^{k}-\frac{\alpha^{k}}{2} \sum_{i=1}^{J} \sum_{j=1}^{J} \phi_{j}^{k}=\sum_{j=1}^{J} n_{j}^{k} \sigma_{i j}^{k} p_{j}+n_{0}^{k},
\end{aligned}
$$

where $\mu_{j}^{k}=\mathbb{E}^{k}\left[X_{j}\right]$ and $\sigma_{i j}^{k}$ represents investor $k$ 's belief of the covariance between $X_{i}$ and $X_{j}$. Note that in the special case when all investors share the same belief and the same margin requirement is imposed on all assets, the optimal portfolio weights of investors with different degrees of risk aversion will be proportional to each other (Ma et al. (2015)).

Let $P=\left(p_{1}, \cdots, p_{J}\right)^{\top}, \Phi^{k}=\left(\phi_{1}^{k}, \cdots, \phi_{J}^{k}\right)^{\top}, N^{k}=\left(n_{1}^{k}, \cdots, n_{J}^{k}\right)^{\top}$, and $\Sigma^{k}$ be a covariance matrix with $(i, j)$ th element $\sigma_{i j}^{k}$. The following definition characterizes the equilibrium price of the market.

Definition 1. A vector $P^{*} \in \Re^{J}$ is called an equilibrium price vector of the market if there exist $\Phi^{1 *}, \cdots, \Phi^{K *} \in \Re^{J}$ such that

(1) $\Phi^{k *}$ solves $\left(I^{k}\right)$ at $P^{*}$ for all $k$;

(2) $\sum_{k=1}^{K} \Phi^{k *}=\sum_{k=1}^{K} N^{k}$

We remark that the existence of the equilibrium can be guaranteed under mild regularity conditions (cf. e.g., Sun and Yang (2003)). Since our goal is to find an equilibrium price vector, we assume throughout this paper that an equilibrium price $P^{*}$ exists and is unique.

We impose the following assumptions on the model.

\section{Assumptions}

A1. $\sum_{k=1}^{K} n_{j}^{k}>0$ for $j=1, \cdots, J$.

A2. The covariance matrix $\Sigma^{k}$ is positive definite for all $k=1, \cdots, K$.

A3. The price vector $P$ takes values from a compact and convex set $\Theta \subseteq \Re_{+}^{J}$.

A4. The equilibrium price vector $P^{*}$ lies in the interior of $\Theta$.

Assumption A1 rules out the possibility of trading primitive and derivative securities characterized by zero-share endowments. It has been shown in Ma et al. (2015) that under such an assumption, 
CAPM with margin rules holds a unique equilibrium when all investors share homogeneous beliefs. Assumptions A2 and A3 ensure that the portfolio selection problem $\left(I^{k}\right)$ is strictly convex and thus has a unique optimal solution for any given $P \in \Theta$. In particular, A2 is a mild condition imposed on the portfolio selection model itself and A3 is a relaxation of the customary condition that asset prices assume non-negative values from bounded sets characterized by box constraints (see, e.g., Sun and Yang (2003); Jofré et al. (2007)). We remark that while assumption A4 is potentially strong, the condition can generally be satisfied in practice when equilibrium prices are strictly positive and the set $\Theta$ is chosen sufficiently large. Sufficient conditions that guarantee the positiveness of equilibrium prices in CAPM can be found in e.g., Nielsen (1992) and Levy (2007).

\section{The Algorithm}

Consider a recursive procedure that generates a sequence of price vectors $\left\{P_{n}\right\}_{n=0}^{\infty}$, where $P_{n}=$ $\left(p_{n, 1}, \ldots, p_{n, J}\right)^{\top}$ is an approximation of the equilibrium price $P^{*}$ obtained at the $n$th iteration. Let $\Phi_{n}^{k *}=\left(\phi_{n, 1}^{k *}, \ldots, \phi_{n, J}^{k *}\right)^{\top}$ be the solution to the optimal portfolio selection problem $\left(I^{k}\right)$ when the initial asset prices are given by $P_{n}$. Intuitively, the sum $\sum_{k=1}^{K} \phi_{n, j}^{k *}$ can be viewed as the market demand for asset $j$ under price $p_{n, j}$, whereas $\sum_{k=1}^{K} n_{j}^{k}$ can be viewed as the market supply of the same asset. If $p_{n, j}$ is lower than the equilibrium price, there will be an excess demand over supply, i.e., $\sum_{k=1}^{K} \phi_{n, j}^{k *}-\sum_{k=1}^{K} n_{j}^{k}>0$. On the other hand, a price $p_{n, j}$ that is above the equilibrium will result in excess supply, leading to a negative difference $\sum_{k=1}^{K} \phi_{n, j}^{k *}-\sum_{k=1}^{K} n_{j}^{k}<0$. Therefore, it seems natural that the price of asset $j$ should be updated at each iteration by adjusting its current value $p_{n, j}$ in the direction of the difference $\sum_{k=1}^{K} \phi_{n, j}^{k *}-\sum_{k=1}^{K} n_{j}^{k}$. This intuition leads to the following iterative formula for updating asset prices:

$$
p_{n+1, j}=p_{n, j}+a_{n}\left(\sum_{k=1}^{K} \phi_{n, j}^{k *}-\sum_{k=1}^{K} n_{j}^{k}\right)
$$

where $a_{n} \in(0,1)$ is a step size/gain parameter that controls the amount of adjustment at each step. We assume that the step size satisfies

$$
\lim _{n \rightarrow \infty} a_{n}=0, \quad \sum_{n=0}^{\infty} a_{n}=\infty, \text { and } \sum_{n=0}^{\infty} a_{n}^{2}<\infty .
$$

Recursion (4) gives rise to our proposed algorithm for finding the equilibrium solution to the model described in Section 2. The major steps of the algorithm are presented below. 


\section{Algorithm 1. Computing a CAPM equilibrium with margin rules}

Step 0: Specify an initial price vector $P_{0} \in \Theta$ and a gain sequence $\left\{\alpha_{k}\right\}$. Set iteration counter $n=0$.

Step 1: Solve the portfolio selection problem $\left(I^{k}\right)$ under $P_{n}$ to obtain $\Phi_{n}^{k *}$ for all $k=1, \ldots, K$.

Step 2: Update the price vector $P_{n+1}=\Pi_{\Theta}\left(P_{n}+a_{n}\left(\sum_{k=1}^{K} \Phi_{n}^{k *}-\sum_{k=1}^{K} N^{k}\right)\right)$.

Step 3: If a specified stopping rule is satisfied, then terminate the algorithm; otherwise set $n=n+1$ and go to Step 1.

Note that, as we will discuss in Section 4, the optimal portfolio selection problem at Step 1 can be equivalently formulated as an unconstrained strictly convex minimization problem. Its unique solution can be efficiently obtained using many standard numerical methods. The price updating formula at Step 2 is essentially a vector version of Equation (4) with an additional projection operator $\Pi_{\Theta}$ that projects an iterate back onto the set $\Theta$ whenever it falls outside of $\Theta$. The operator prevents the iterates of the algorithm from becoming too large, e.g., due to inappropriate choices of the gain $a_{n}$, and ensures the sequence $\left\{P_{n}\right\}$ to stay bounded as the search proceeds. Since we are primarily interested in analyzing the asymptotic convergence of the algorithm, we have not specified a stopping rule. In practice, the algorithm can be terminated either when a given computing budget is exhausted or when the differences $\left|\sum_{k=1}^{K} \phi_{n, j}^{k *}-\sum_{k=1}^{K} n_{j}^{k}\right|$ fall below a predetermined tolerance $\epsilon>0$ for all $j$.

\section{Convergence Analysis}

The decision variable $\phi_{0}^{k}$ appearing in the constraint of $\left(I^{k}\right)$ can be expressed in terms of variables $\phi_{j}^{k-}$ and $\phi_{j}^{k+}, j=1, \ldots, J$ as follows:

$$
\phi_{0}^{k}=\sum_{j=1}^{J} n_{j}^{k} p_{j}+n_{0}^{k}-\sum_{j=1}^{J}\left(m_{j}^{k} \phi_{j}^{k-}+\phi_{j}^{k+}\right) p_{j}
$$

Substituting (6) into the objective function in $\left(I^{k}\right)$ and removing terms that are constant with respect to $\phi_{j}^{k}$, we arrive at the following equivalent optimization problem:

$$
\min _{\phi_{j}^{k}, j=1, \ldots, J} \frac{\alpha^{k}}{2} \sum_{i=1}^{J} \sum_{j=1}^{J} \phi_{j}^{k} \phi_{i}^{k} \sigma_{i j}^{k}-\sum_{j=1}^{J}\left[\left(\mu_{j}^{k}-\left(1-r m_{j}^{k}\right) p_{j}\right) \phi_{j}^{k}-r\left(m_{j}^{k}+1\right) p_{j} \phi_{j}^{k+}\right] .
$$


Note that under assumptions A2 and A3, the objective function in (7) is continuous and strictly convex. Consequently, for any given $P \in \Theta$, the corresponding optimal solution to (7), denoted by $\Phi^{k}(P)$, is unique and can be viewed as a function of the price vector $P$. However, since the term $\phi_{j}^{k+}$ is not differentiable with respect to $\phi_{j}^{k}$ at the origin, obtaining an explicit closed-form representation for $\Phi^{k}(P)$ can be difficult.

We begin our convergence analysis by establishing some important properties of the portfolio selection function $\Phi^{k}(P)$. Let $\|\cdot\|$ be the usual Euclidean norm, $m^{k}=\left(m_{1}^{k}, \ldots, m_{J}^{k}\right)^{\top}$, and $\mathbb{1}$ be a vector of all ones with appropriate dimension. Define $\Lambda^{k}=\operatorname{diag}\left(\mathbb{1}-r m^{k}\right)$ and $\Gamma^{k}=\operatorname{diag}\left(\mathbb{1}+m^{k}\right)$, where for a given column vector $\mathbf{v}, \operatorname{diag}(\mathbf{v})$ signifies a diagonal matrix with its diagonal elements given by the entries in v. The following lemma, due to Cottle et al. (1992) and Yen (1995), provides a useful characterization of the solutions to parametric quadratic programming problems.

Lemma 1. (Cottle et al. (1992) and Yen (1995)) Consider the following quadratic programming problem parameterized by two vectors $c \in \Re^{n}$ and $\theta \in \Re^{r}$ :

$$
\begin{aligned}
& \min \frac{1}{2} x^{\top} D x+c^{\top} x \\
& \text { subject to } A x \geq \theta, x \geq 0,
\end{aligned}
$$

where $D$ is a symmetric positive definite matrix and $A$ is an $r \times n$ real matrix. Define $K(\theta)=\{x \in$ $\left.\Re^{n}: A x \geq \theta, x \geq 0\right\}$ and $\Lambda=\left\{\theta \in \Re^{r} \mid K(\theta) \neq \emptyset\right\}$. For given $c \in \Re^{n}$ and $\theta \in \Lambda$, denote by $x(c, \theta)$ the unique optimal solution to (8). There exists a constant $L>0$ depending on $D$ and $A$ such that

$$
\left\|x\left(c^{\prime}, \theta^{\prime}\right)-x(c, \theta)\right\| \leq L\left(\left\|c^{\prime}-c\right\|+\left\|\theta^{\prime}-\theta\right\|\right)
$$

for all $\theta, \theta^{\prime} \in \Lambda$ and $c, c^{\prime} \in \Re^{n}$.

The above lemma, when applied to problem (7), implies that $\Phi^{k}(P)$ is Lipschitz continuous in the price vector $P$.

Proposition 1. If Assumptions A2 and A3 hold, then there exists a constant $L_{k}>0$ such that for any $P, \tilde{P} \in \Theta,\left\|\Phi^{k}(P)-\Phi^{k}(\tilde{P})\right\| \leq L_{k}\|P-\tilde{P}\|$.

Proof. Let $\psi_{j}^{k}=\phi_{j}^{k+}$ and $\Psi^{k}=\left(\psi_{1}^{k}, \ldots, \psi_{J}^{k}\right)^{\top}$. Rewrite $\left(I^{k}\right)$ in the vector form

$$
\begin{aligned}
& \min _{\Phi^{k}, \Psi^{k} \in \Re^{J}} \frac{\alpha^{k}}{2}\left(\Phi^{k}\right)^{\top} \Sigma^{k} \Phi^{k}+r \cdot P^{\top} \cdot \Gamma^{k} \cdot \Psi^{k}-\left(\mu^{k}-\Lambda^{k} \cdot P\right)^{\top} \cdot \Phi^{k} \\
& \text { subject to } \Psi^{k} \geq \Phi^{k}, \Psi^{k} \geq 0 .
\end{aligned}
$$


Let $\lambda^{k}$ be the Lagrange multipliers associated with the constraints $\Psi^{k} \geq \Phi^{k}$. Then we obtain the following Lagrange function:

$$
L^{k}\left(\Phi^{k}, \Psi^{k} ; \lambda^{k}\right)=\frac{\alpha^{k}}{2}\left(\Phi^{k}\right)^{\top} \Sigma^{k} \Phi^{k}+r P^{\top} \cdot \Gamma^{k} \cdot \Psi^{k}-\left(\mu^{k}-\Lambda^{k} \cdot P\right)^{\top} \cdot \Phi^{k}+\left(\lambda^{k}\right)^{\top} \cdot\left(\Phi^{k}-\Psi^{k}\right) .
$$

Thus, the dual program of (9) can be written as

$$
\max _{\lambda^{k} \geq 0} \min _{\Phi^{k} \in \Re^{J}, \Psi^{k} \geq 0} L^{k}\left(\Phi^{k}, \Psi^{k} ; \lambda^{k}\right) .
$$

Specifically, we have

$$
\begin{aligned}
& \min _{\Phi^{k} \in \Re^{J}, \Psi^{k} \geq 0} L^{k}\left(\Phi^{k}, \Psi^{k} ; \lambda^{k}\right) \\
& =\min _{\Phi^{k} \in \Re^{J}, \Psi^{k} \geq 0}\left\{\frac{\alpha^{k}}{2}\left(\Phi^{k}\right)^{\top} \Sigma^{k} \Phi^{k}+r P^{\top} \cdot \Gamma^{k} \cdot \Psi^{k}-\left(\mu^{k}-\Lambda^{k} \cdot P\right)^{\top} \cdot \Phi^{k}+\left(\lambda^{k}\right)^{\top} \cdot\left(\Phi^{k}-\Psi^{k}\right)\right\} \\
& =\min _{\Phi^{k} \in \Re^{J}}\left\{\frac{\alpha^{k}}{2}\left(\Phi^{k}\right)^{\top} \Sigma^{k} \Phi^{k}-\left(\mu^{k}-\Lambda^{k} \cdot P-\lambda^{k}\right)^{\top} \cdot \Phi^{k}\right\}+\min _{\Psi^{k} \geq 0}\left(r \Gamma^{k} \cdot P-\lambda^{k}\right)^{\top} \cdot \Psi^{k} .
\end{aligned}
$$

For any given $\lambda_{k}$, the first minimization problem in (10) can be solved analytically by setting the first-order derivatives to zero, yielding

$$
\Phi^{k}(P)=\frac{1}{\alpha^{k}}\left(\Sigma^{k}\right)^{-1} \cdot\left(\mu^{k}-\Lambda^{k} \cdot P-\lambda^{k}\right)
$$

On the other hand, the optimal value of the second minimization problem in (10) is given by

$$
\min _{\Psi^{k} \geq 0}\left\{\left(r \Gamma^{k} \cdot P-\lambda^{k}\right)^{\top} \Psi^{k}\right\}= \begin{cases}0 & \lambda^{k} \leq r \Gamma^{k} \cdot P \\ -\infty & \text { otherwise }\end{cases}
$$

Substituting (11) and (12) into $L^{k}\left(\Phi^{k}, \Psi^{k} ; \lambda^{k}\right)$, it can be seen that the dual program of (9) is equivalent to the following minimization problem:

$$
\begin{aligned}
& \left(D^{k}\right) \quad \min \left(\mu^{k}-\Lambda^{k} \cdot P-\lambda^{k}\right)^{\top} \cdot\left(\Sigma^{k}\right)^{-1} \cdot\left(\mu^{k}-\Lambda^{k} \cdot P-\lambda^{k}\right) \\
& \text { s.t. } 0 \leq \lambda^{k} \leq r \Gamma^{k} \cdot P .
\end{aligned}
$$

The above problem can be cast in the form of (8) by taking $x=\lambda^{k}, D=\left(\Sigma^{k}\right)^{-1}, c=-2\left(\Sigma^{k}\right)^{-1}\left(\mu^{k}-\right.$ $\left.\Lambda^{k} \cdot P\right), A=-\operatorname{diag}(\mathbb{1})$, and $\theta=-r \Gamma^{k} \cdot P$. For a given $P$, let $\lambda^{k}(P)$ be an optimal solution to $\left(D^{k}\right)$. 
Clearly, by Assumption A2, $\lambda^{k}(P)$ is unique. Therefore, all conditions in Lemma 1 are satisfied and we conclude that there exists a constant $l_{k}>0$ depending on $D$ and $A$ such that for any $P, \tilde{P} \in \Theta$,

$$
\begin{aligned}
\left\|\lambda^{k}(P)-\lambda^{k}(\tilde{P})\right\| & \leq l_{k} \cdot(\|c-\tilde{c}\|+\|\theta-\tilde{\theta}\|) \\
& =l_{k} \cdot\left(2\left\|\left(\Sigma^{k}\right)^{-1} \Lambda^{k}(P-\tilde{P})\right\|+r\left\|\Gamma^{k}(P-\tilde{P})\right\|\right) \\
& \leq l_{k} \cdot\left(2\left\|\left(\Sigma^{k}\right)^{-1} \Lambda^{k}\right\|+r\left\|\Gamma^{k}\right\|\right)\|P-\tilde{P}\| \\
& =d_{k}\|P-\tilde{P}\|,
\end{aligned}
$$

where $\tilde{c}$ and $\tilde{\theta}$ are the respective values of $c$ and $\theta$ when $P$ is replaced by $\tilde{P}$, and $d_{k}=c_{k}$. $\left(2\left\|\left(\Sigma^{k}\right)^{-1} \Lambda^{k}\right\|+r\left\|\Gamma^{k}\right\|\right)$. By Assumptions A2 and A3, $\left(I^{k}\right)$ and $\left(D^{k}\right)$ are both strictly convex optimization problems. Thus, the strong duality theorem implies that their corresponding optimal solutions $\Phi^{k}(P)$ and $\lambda^{k}(P)$ satisfy Equation (11). Consequently, we obtain

$$
\begin{aligned}
\left\|\Phi^{k}(P)-\Phi^{k}(\tilde{P})\right\| & =\left\|\frac{1}{\alpha^{k}}\left(\Sigma^{k}\right)^{-1} \cdot\left[-\Lambda^{k}(P-\tilde{P})-\left(\lambda^{k}(P)-\lambda^{k}(\tilde{P})\right)\right]\right\| \\
& \leq \frac{1}{\alpha^{k}}\left\|\left(\Sigma^{k}\right)^{-1}\right\| \cdot\left(\left\|\Lambda^{k}\right\| \cdot\|P-\tilde{P}\|+\left\|\lambda^{k}(P)-\lambda^{k}(\tilde{P})\right\|\right) \\
& \leq \frac{1}{\alpha^{k}}\left\|\left(\Sigma^{k}\right)^{-1}\right\| \cdot\left(\left\|\Lambda^{k}\right\|+d_{k}\right) \cdot\|P-\tilde{P}\| .
\end{aligned}
$$

Hence the proof is completed by setting $L_{k}=\frac{1}{\alpha^{k}}\left\|\left(\Sigma^{k}\right)^{-1}\right\| \cdot\left(\left\|\Lambda^{k}\right\|+d_{k}\right)$.

Intuitively speaking, since the new price vector $P_{n+1}$ is updated at Step 2 of the algorithm in an incremental fashion based on $P_{n}$ (due to the step size parameter $a_{n}$ ), Proposition 1 implies that the portfolio selection function at $P_{n+1}$, i.e., $\Phi_{n+1}^{k *}=\Phi^{k}\left(P_{n+1}\right)$, will not deviate significantly from $\Phi_{n}^{k *}$. This ensures the stability of the sequence of price vectors generated by the algorithm, i.e., no big jump in $\left\{P_{n}\right\}$ occurs as iteration goes on.

The next result partially characterizes the optimal solution to $(7)$ and shows that $\Phi^{k}(P)$ is continuous and piecewise linear in the price vector $P$.

Lemma 2. If Assumptions A2 and A3 hold, then $\Phi^{k}(P)$ is a continuous and piecewise linear function on $\Theta$.

Proof. Since the value of $\phi_{j}^{k+}$ is determined by the sign of $\phi_{j}^{k}$, we partition the entire feasible region $\Re^{J}$ of $\Phi^{k}(P)$ into $2^{J}$ subregions of the form $S_{t} \triangleq \underbrace{S_{t 1} \times \cdots \times S_{t i} \cdots \times S_{t J}}_{J}$ for $t=1, \cdots, 2^{J}$, where for each $i=1, \ldots, J, S_{t i}$ either equals $\Re_{+} \cup\{0\}$ or $\Re_{-}$. The closure, interior, and the boundary 
of $S_{t}$ are denoted by $c l\left(S_{t}\right), \operatorname{int}\left(S_{t}\right)$, and $\partial\left(S_{t}\right)$, respectively. Let $\theta_{t}^{k}=\left\{P \mid \Phi^{k}(P) \in S_{t}, P \in \Theta\right\}$. Obviously, due to the uniqueness of $\Phi^{k}(P)$ under Assumptions A2 and A3, we have $\Theta=\cup_{t=1}^{2^{J}} \theta_{t}^{k}$ and $\theta_{i}^{k} \cap \theta_{j}^{k}=\emptyset$ for all $i \neq j$.

Consider optimization problem (7) restricted to the closure of $S_{t}$, i.e.,

$$
\begin{aligned}
\left(I^{k t}\right): \quad & \min _{\Phi^{k} \in R^{J}} \frac{\alpha^{k}}{2}\left(\Phi^{k}\right)^{\top} \Sigma^{k} \Phi^{k}-\left(\mu^{k}-\Lambda^{k} \cdot P\right)^{\top} \cdot \Phi^{k}+r \cdot P^{\top}\left(\Gamma^{k} \cdot U_{t}^{k}\right) \cdot \Phi^{k} \\
& \text { subject to } \Phi^{k} \in \operatorname{cl}\left(S_{t}\right),
\end{aligned}
$$

where $U_{t}^{k}=\operatorname{diag}\left(\mathbb{1}_{t}^{k}\right)$ and $\mathbb{1}_{t}^{k}$ is a vector of indices with its $j$ th element $\mathbb{1}_{t j}^{k}$ given by

$$
\mathbb{1}_{t j}^{k}=\left\{\begin{array}{l}
1, \quad S_{t j}=\Re_{+} \cup\{0\} \\
0, \quad S_{t j}=\Re_{-} .
\end{array}\right.
$$

Since $\left(I^{k t}\right)$ is quadratic and strictly convex, it has a unique optimal solution $\Phi^{k t}(P)$ with its $j$ th component located either in the interior $\operatorname{int}\left(S_{t j}\right)$ or on the boundary $\partial\left(S_{t j}\right)$. The components of $\Phi^{k t}(P)$ that lie on the boundary must take the value 0 , whereas those that lie in the interior satisfy the first order necessary condition for optimality and can be obtained analytically by setting partial derivatives to zero. Let $M=\{1, \cdots, J\}$ and denote by $\mathbb{M}$ the collection of all subsets of $M$. It follows that there must exist sets $I \in \mathbb{M}$ and $I^{c}=M \backslash I$ such that

$$
\Phi_{I}^{k t}(P)=\frac{1}{\alpha^{k}}\left(\sum_{I}^{k}\right)^{-1} \cdot\left[\mu_{I}^{k}-\left(\Lambda_{I}^{k}+r \Gamma_{I}^{k} \cdot U_{I}^{k t}\right) \cdot P_{I}\right]
$$

and $\Phi_{I^{c}}^{k t}(P)=0$, where $\Phi_{I}^{k t}=\left(\phi_{u}^{k t}(P), u \in I\right)^{\top}$ and $\Phi_{I^{c}}^{k t}=\left(\phi_{v}^{k t}(P), v \in I^{c}\right)^{\top}$ are vectors consisting of elements in $\Phi^{k t}(P)$ with indices belonging to $I$ and $I^{c}$, respectively, and $\mu_{I}^{k}, P_{I}, \Sigma_{I}^{k}, \Lambda_{I}^{k}, \Gamma_{I}^{k}, U_{I}^{k t}$ are the corresponding subvectors and principal submatrices constructed in the same way. Note that $I=\emptyset$ indicates $\Phi^{k t}(P)=0$ and $I^{c}=\emptyset$ indicates $\Phi^{k t}(P) \in \operatorname{int}\left(S_{t}\right)$.

Label the elements of $\mathbb{M}$ as $I_{i}, i=1, \ldots, 2^{J}$. For a given index set $I_{i} \in \mathbb{M}$, let $\vartheta_{i}^{k t}=\{P \in$ $\left.\Theta \mid \phi_{u}^{k t}(P) \neq 0, \phi_{v}^{k t}(P)=0, \forall u \in I_{i}, \forall v \in M \backslash I_{i}\right\}$. It is easy to verify that $\left\{\vartheta_{i}^{k t}\right\}_{i=1}^{2^{J}}$ forms a partition of $\Theta$, i.e. $\Theta=\cup_{i=1}^{2^{J}} \vartheta_{i}^{k t}$ and $\vartheta_{i}^{k t} \cap \vartheta_{j}^{k t}=\emptyset$ whenever $i \neq j$. Combining this with the partition $\left\{\theta_{t}^{k}\right\}_{t=1}^{2^{J}}$ defined earlier, we can write $\Theta$ as the union of $4^{J}$ disjoint subregions, $\Theta=\cup_{t, i=1}^{2^{J}}\left(\theta_{t}^{k} \cap \vartheta_{i}^{k t}\right)$. Therefore, for any given price vector $P \in \Theta$, it must be located in one of these (non-empty) $4^{J}$ subregions, over which the solution $\Phi^{k}(P)$ to $(7)$ can be expressed in the linear form of (13). This observation, together with Proposition 1 , shows that $\Phi^{k}(P)$ is a continuous piecewise linear function 
of $P$ over $\Theta$.

In addition, as shown in the following lemma, when the price vector $P$ falls in a certain range, the value of $\Phi^{k}(P)$ will remain identically zero.

Lemma 3. $\Phi^{k}(P)=0$ if and only if $\Lambda^{k} \cdot P \leq \mu^{k} \leq(1+r) \cdot P$.

Proof. Since $\left(I^{k}\right)$ and $\left(D^{k}\right)$ are strictly convex (see the proof of Proposition 1), the strong duality theorem implies that their corresponding optimal solutions $\Phi^{k}$ and $\lambda^{k *}$ satisfy Equation (11). We see that if $\Lambda^{k} \cdot P \leq \mu^{k} \leq(1+r) P$, then the optimal solution to $\left(D^{k}\right)$ is given by $\lambda^{k *}=\mu^{k}-\Lambda^{k} \cdot P$. This in turn implies $\Phi^{k}(P)=0$. Conversely, if $\Phi^{k}(P)=0$, then it follows from (11) that $\lambda^{k *}=\mu^{k}-\Lambda^{k} \cdot P$. Finally, since $\lambda^{k *}$ satisfies the constraint of $\left(D^{k}\right)$, we must have $\Lambda^{k} \cdot P \leq \mu^{k} \leq(1+r) P$. This completes the proof of the lemma.

The result of Lemma 3 is consistent with existing findings in the literature; see, e.g., Figure 2 in Anufriev and Tuinstra (2013) for a simple illustration when the market only consists of a risky and a risk-free assets and Ma et al. (2015) for an analysis of this effect when the covariance matrix $\Sigma^{k}$ is diagonal. Intuitively, if an asset $j$ satisfies the inequality $\left(1-r m_{j}^{k}\right) \cdot P_{j} \leq \mu_{j}^{k}$ (i.e., the $j$-th inequality in $\Lambda^{k} \cdot P \leq \mu^{k}$, then short selling a unit of asset $j$ will yield a return of $P_{j}-\mu_{j}^{k}$, which is less than $r m_{j}^{k} P_{j}$, the interest earned by instead depositing the funds required for marginal cost into a bank. Thus, short selling asset $j$ is apparently undesirable. Similarly, if $\mu_{j}^{k} \leq(1+r) P_{j}$, then the return $\mu_{j}^{k}-P_{j}$ resulting from buying a unit of asset $j$ will be less than the interest earned $r P_{j}$ by instead depositing the money required for purchasing the asset into a bank. Thus, there is no incentive in buying asset $j$.

For a given non-empty index set $I \in \mathbb{M}$, consider the matrix $\left(\Sigma_{I}^{k}\right)^{-1}\left(\Lambda_{I}^{k}+r \Gamma_{I}^{k} \cdot U_{I}^{k t}\right)$, where $\Sigma_{I}^{k}$, $\Lambda_{I}^{k}, \Gamma_{I}^{k}$, and $U_{I}^{k t}$ are submatrices constructed by taking rows and columns specified in $I$ from the respective matrices $\Sigma^{k}, \Lambda^{k}, \Gamma^{k}$, and $U^{k t}$ (see the proof of Lemma 2). Note that since $\Lambda_{I}^{k}+r \Gamma_{I}^{k} \cdot U_{I}^{k t}$ is a diagonal matrix with its $i$ th diagonal element given by

$$
1-r m_{i}^{k}+r\left(m_{i}^{k}+1\right) \cdot \mathbb{1}_{i}^{k t}= \begin{cases}1-r m_{i}^{k}, & \mathbb{1}_{i}^{k t}=0 \\ 1+r, & \mathbb{1}_{i}^{k t}=1\end{cases}
$$

it can be rewritten as $\mathbb{I}+r C_{I}^{k t}$, where $\mathbb{I}$ is the identity matrix and $C_{I}^{k t}$ is a diagonal matrix with its diagonal elements either being $-m_{j}^{k}$ (for some $j$ ) or 1 . Let $\lambda_{\min }^{k}$ be the smallest eigenvalue of $\left(\Sigma^{k}\right)^{-1}$ and $\tilde{\lambda}_{I}^{k t}$ be the smallest eigenvalue of $\frac{C_{I}^{k t}\left(\Sigma_{I}^{k}\right)^{-1}+\left(\Sigma_{I}^{k}\right)^{-1} C_{I}^{k t}}{2}$. Define $\tilde{\lambda}_{\min }^{k}=\min _{I \in \mathbb{M}, I \neq \emptyset, t=1, \ldots, 2^{J}} \tilde{\lambda}_{I}^{k t}$. 
The following result provides a sufficient condition to ensure the positive definiteness of the matrix $\left(\Sigma_{I}^{k}\right)^{-1}\left(\Lambda_{I}^{k}+r \Gamma_{I}^{k} \cdot U_{I}^{k t}\right)$.

Lemma 4. If $\min _{k=1, \ldots, K}\left(\lambda_{\text {min }}^{k}+r \tilde{\lambda}_{\text {min }}^{k}\right)>0$, then $\left(\Sigma_{I}^{k}\right)^{-1}\left(\Lambda_{I}^{k}+r \Gamma_{I}^{k} \cdot U_{I}^{k t}\right)$ is positive definite for all nonempty sets $I \in \mathbb{M}, t=1, \ldots, 2^{J}$, and $k=1, \cdots, K$.

Proof. For a given nonempty index set $I \in \mathbb{M}$, since $\Sigma_{I}^{k}$ is a principal submatrix of the symmetric matrix $\Sigma^{k}$, by Cauchy interlace theorem (cf. e.g., Chapter 4 in Horn and Johnson (2012)), its largest eigenvalue is dominated by the largest eigenvalue of $\Sigma^{k}$. Therefore, the smallest eigenvalue of $\left(\Sigma_{I}^{k}\right)^{-1}$ is lower bounded by $\lambda_{\min }^{k}$.

Let $x \neq 0$ be any given vector of appropriate dimension. It follows that

$$
\begin{aligned}
x^{\top}\left(\Sigma_{I}^{k}\right)^{-1}\left(\Lambda_{I}^{k}+r \Gamma_{I}^{k} \cdot U_{I}^{k t}\right) x & =x^{\top}\left(\Sigma_{I}^{k}\right)^{-1}\left(\mathbb{I}+r C_{I}^{k t}\right) x \\
& =x^{\top}\left(\Sigma_{I}^{k}\right)^{-1} x+r x^{\top}\left(\Sigma_{I}^{k}\right)^{-1} C_{I}^{k t} x \\
& =x^{\top}\left(\Sigma_{I}^{k}\right)^{-1} x+r x^{\top} \frac{\left(\Sigma_{I}^{k}\right)^{-1} C_{I}^{k t}+C_{I}^{k t}\left(\Sigma_{I}^{k}\right)^{-1}}{2} x \\
& \geq\left(\lambda_{\text {min }}^{k}+r \tilde{\lambda}_{I}^{k t}\right)\|x\|^{2} \\
& \geq\left(\lambda_{\text {min }}^{k}+r \tilde{\lambda}_{\text {min }}^{k}\right)\|x\|^{2} .
\end{aligned}
$$

Thus, by assumption, we have $x^{\top}\left(\sum_{I}^{k}\right)^{-1}\left(\Lambda_{I}^{k}+r \Gamma_{I}^{k} \cdot U_{I}^{k t}\right) x>0$. This shows that $\left(\sum_{I}^{k}\right)^{-1}\left(\Lambda_{I}^{k}+r \Gamma_{I}^{k} \cdot U_{I}^{k t}\right)$ is positive definite.

Lemma 4 leads to the following result, which indicates that the excess demands across all investors, $\sum_{k=1}^{K} \Phi^{k}(P)-\sum_{k=1}^{K} N^{k}$, will react in the opposite direction with respect to price changes around the equilibrium prices $P^{*}$.

Lemma 5. If Assumptions A1-A4 hold and $r$ satisfies $\min _{k=1, \ldots, K}\left(\lambda_{\text {min }}^{k}+r \tilde{\lambda}_{\text {min }}^{k}\right)>0$, then there exists a constant $h<0$ such that $\left(\sum_{k=1}^{K} \Phi^{k}(P)-\sum_{k=1}^{K} N^{k}\right)^{\top}\left(P-P^{*}\right) \leq h \cdot\left\|P-P^{*}\right\|^{2}$ for all $P \in \Theta$.

Proof. The result holds trivially when $P=P^{*}$, so we consider the case $P \neq P^{*}$. For a fixed investor $k$, let $\left\{\theta_{t}^{k}\right\}_{t=1}^{2^{J}}$ and $\left\{\vartheta_{i}^{k t}\right\}_{i=1}^{2^{J}}$ be the two partitions defined in the proof of Lemma 2. We know that there must exist a $t^{*}$ and an index set $I_{i_{k}} \subseteq \mathbb{M}$ such that $P^{*} \in \Omega_{i_{k}}^{k t^{*}}=\theta_{t^{*}}^{k} \cap \vartheta_{i_{k}}^{k t^{*}}$. By Lemma 3 and the definition of $\vartheta_{i_{k}}^{k t}, \Omega_{i_{k}}^{k t^{*}}$ is either contained in $\Theta \cap\left\{P \mid \Lambda^{k} \cdot P \leq \mu^{k} \leq(1+r) \cdot P\right\}$ or in its complement. If $\Omega_{i_{k}}^{k t^{*}} \subseteq \Theta \cap\left\{P \mid \Lambda^{k} \cdot P \leq \mu^{k} \leq(1+r) \cdot P\right\}$, then Lemma 3 indicates that $\Phi^{k}\left(P^{*}\right)=0$ and $I_{i_{k}}=\emptyset$; otherwise, the set $I_{i_{k}}$ contains at least one index $j$ such that $\phi_{j}^{k}\left(P^{*}\right) \neq 0$. 
For any price vector $P \in \Theta$, without loss of generality, we assume that $P \in \Omega_{p}^{k t}=\theta_{t}^{k} \cap \vartheta_{p}^{k t}$ for some $t \in\left\{1, \ldots, 2^{J}\right\}$ and index set $I_{p} \in \mathrm{M}$. We consider the following three cases: (1) $\Omega_{p}^{k t}=\Omega_{i_{k}}^{k t^{*}}$; (2) $\Omega_{p}^{k t} \cap \Omega_{i_{k}}^{k t^{*}}=\emptyset$ and $P^{*} \in c l \Omega_{i_{k}}^{k t^{*}} \cap c l \Omega_{p}^{k t}$; (3) $\Omega_{p}^{k t} \cap \Omega_{i_{k}}^{k t^{*}}=\emptyset$ and $P^{*} \notin c l \Omega_{i_{k}}^{k t^{*}} \cap c l \Omega_{p}^{k t}$.

In Case (1), $P^{*}$ and $P$ lie in the same subregion. If $\Omega_{p}^{k t}=\Omega_{i_{k}}^{k t^{*}} \subseteq \Theta \cap\left\{P \mid \Lambda^{k} \cdot P \leq \mu^{k} \leq(1+r) \cdot P\right\}$, then $\Phi^{k}(P)=\Phi^{k}\left(P^{*}\right)=0$, in which case we have $\left\langle\Phi^{k}(P)-\Phi^{k}\left(P^{*}\right), P-P^{*}\right\rangle=0$. Otherwise, if $\Omega_{i_{k}}^{k t *} \nsubseteq \Theta \cap\left\{P \mid \Lambda^{k} \cdot P \leq \mu^{k} \leq(1+r) \cdot P\right\}$, then the index set $I_{i_{k}}$ (and hence $I_{p}$ ) is nonempty and both $\Phi^{k}(P)$ and $\Phi^{k}\left(P^{*}\right)$ can be expressed in the form of (13):

$$
\Phi_{I_{i_{k}}}^{k t}(P)=\frac{1}{\alpha^{k}}\left(\sum_{I_{i_{k}}}^{k}\right)^{-1} \cdot\left[\mu_{I_{i_{k}}}^{k}-\left(\Lambda_{I_{i_{k}}}^{k}+r \Gamma_{I_{i_{k}}}^{k} \cdot U_{I_{i_{k}}}^{k t}\right) \cdot P_{I_{i_{k}}}\right] \text { and } \Phi_{I_{i_{k}}^{c}}^{k t}(P)=0 .
$$

It follows that

$$
\begin{aligned}
\left\langle\Phi^{k}(P)-\Phi^{k}\left(P^{*}\right), P-P^{*}\right\rangle & =-\frac{1}{\alpha^{k}}\left(P_{I_{i_{k}}}-P_{I_{i_{k}}}^{*}\right)^{\top} \cdot\left[\left(\Sigma_{I_{i_{k}}}^{k}\right)^{-1} \cdot\left(\Lambda_{I_{i_{k}}}^{k}+r \Gamma_{I_{i_{k}}}^{k} \cdot U_{I_{i_{k}}}^{k t}\right)\right]^{\top} \cdot\left(P_{I_{i_{k}}}-P_{I_{i_{k}}}^{*}\right) \\
& \leq-\frac{1}{\alpha^{k}} \frac{\lambda_{I_{i_{k}}}^{k t}}{2}\left\|P_{I_{i_{k}}}-P_{I_{i_{k}}}^{*}\right\|^{2} \\
& \leq-\frac{1}{\alpha^{k}} \frac{\lambda_{m i n}^{k t}}{2}\left\|P_{I_{i_{k}}}-P_{I_{i_{k}}}^{*}\right\|^{2}
\end{aligned}
$$

where $\lambda_{I_{i_{k}}}^{k t}>0$ is the minimum eigenvalue of the positive definite matrix $\left(\Sigma_{I_{i_{k}}}^{k}\right)^{-1} \cdot\left(\Lambda_{I_{i_{k}}}^{k}+r \Gamma_{I_{i_{k}}}^{k} \cdot U_{I_{i_{k}}}^{k t}\right)$ (cf. Lemma 4) and $\lambda_{\text {min }}^{k t}=\min _{I_{i_{k}} \in \mathbb{M}, I_{i_{k}} \neq \emptyset} \lambda_{I_{i_{k}}}^{k t}$.

Case (2) implies that $P^{*}$ lies on the boundary of the subregion containing $P$. By the continuity of $\Phi^{k}(P)$ (cf. Lemma 2), if $I_{p}=\emptyset$ (i.e., $\Omega_{p}^{k t} \subseteq \Theta \cap\left\{P \mid \Lambda^{k} \cdot P \leq \mu^{k} \leq(1+r) \cdot P\right\}$ ), then we must have $I_{i_{k}}=\emptyset$, which shows that $\left\langle\Phi^{k}(P)-\Phi^{k}\left(P^{*}\right), P-P^{*}\right\rangle=0$. On the other hand, if $I_{p} \neq \emptyset$, then Lemma 2 implies that both $\Phi^{k}(P)$ and $\Phi^{k}\left(P^{*}\right)$ will have the form of (13). Consequently, the inner product $\left\langle\Phi^{k}(P)-\Phi^{k}\left(P^{*}\right), P-P^{*}\right\rangle$ satisfies the same inequality (14) with $I_{p}$ replacing $I_{i_{k}}$, i.e., $\left\langle\Phi^{k}(P)-\Phi^{k}\left(P^{*}\right), P-P^{*}\right\rangle \leq-\frac{1}{\alpha^{k}} \frac{\lambda_{\min }^{k t}}{2}\left\|P_{I_{p}}-P_{I_{p}}^{*}\right\|^{2}$. In addition, by Proposition 1 , it is easy to see that $I_{i_{k}} \subseteq I_{p}$. This further shows $\left\langle\Phi^{k}(P)-\Phi^{k}\left(P^{*}\right), P-P^{*}\right\rangle \leq-\frac{1}{\alpha^{k}} \frac{\lambda_{\text {min }}^{k t}}{2}\left\|P_{I_{i_{k}}}-P_{I_{i_{k}}}^{*}\right\|^{2}$. Thus, by noting that $I_{i_{k}} \neq \emptyset$ implies $I_{p} \neq \emptyset$, we obtain the following result under Case (2):

$$
\left\langle\Phi^{k}(P)-\Phi^{k}\left(P^{*}\right), P-P^{*}\right\rangle \begin{cases}\leq 0 & \text { if } I_{i_{k}}=\emptyset \\ \leq-\frac{1}{\alpha^{k}} \frac{\lambda_{\text {min }}^{k t}}{2}\left\|P_{I_{i_{k}}}-P_{I_{i_{k}}}^{*}\right\|^{2} & \text { if } I_{i_{k}} \neq \emptyset\end{cases}
$$

In Case (3), since the subregions $\Omega_{p}^{k t}$ and $\Omega_{i_{k}}^{k t^{*}}$ are disjoint and $P^{*} \notin c l \Omega_{i_{k}}^{k t^{*}} \cap c l \Omega_{j}^{k t}$, there exists $\delta_{k t}>0$ such that $\inf _{P^{\prime} \in c l \Omega_{p}^{k t}}\left\|P^{\prime}-P^{*}\right\| \geq \delta_{k t}$. Thus, by the convexity of $\Theta$ in Assumption A3, the 
line segment $L_{P P^{*}}$ connecting $P$ and $P^{*}$ is also within $\Theta$ and passes either through the common boundary between $\Omega_{p}^{k t}$ and $\Omega_{i_{k}}^{k t^{*}}$ or through boundaries of subregions that separate $\Omega_{p}^{k t}$ and $\Omega_{i_{k}}^{k t^{*}}$.

When $L_{P P^{*}}$ intersects the common boundary of $\Omega_{p}^{k t}$ and $\Omega_{i_{k}}^{k t^{*}}$, it is clear that there exist a vector $\tilde{P} \in \operatorname{cl}\left(\Omega_{i_{k}}^{k t^{*}}\right) \cap \operatorname{cl}\left(\Omega_{p}^{k t}\right)$ and a constant $\tau_{p}^{k t} \in(0,1]$ such that $\tilde{P}=\tau_{p}^{k t} P+\left(1-\tau_{p}^{k t}\right) P^{*}$. Note that the $\tau_{p}^{k t}=1$ case implies $P=\tilde{P}$, so $P$ lies on the boundary of the region containing $P^{*}$ and we obviously have

$$
\left\langle\Phi^{k}(P)-\Phi^{k}\left(P^{*}\right), P-P^{*}\right\rangle \begin{cases}=0 & \text { if } I_{i_{k}}=\emptyset \\ \leq-\frac{1}{\alpha^{k}} \frac{\lambda_{\text {min }}^{k t}}{2}\left\|P_{I_{i_{k}}}-P_{I_{i_{k}}}^{*}\right\|^{2} & \text { if } I_{i_{k}} \neq \emptyset .\end{cases}
$$

If $\tau_{p}^{k t} \in(0,1)$ and $I_{i_{k}}=\emptyset$, then again by the continuity of $\Phi^{k}(P)$, we must have $\Phi^{k}(\tilde{P})=0$. It follows that

$$
\begin{aligned}
\left\langle\Phi^{k}(P)-\Phi^{k}\left(P^{*}\right), P-P^{*}\right\rangle & =\left\langle\Phi^{k}(P)-\Phi^{k}(\tilde{P}), P-P^{*}\right\rangle \\
& =\left\langle\Phi^{k}(P)-\Phi^{k}(\tilde{P}), P-\frac{\tilde{P}-\tau_{p}^{k t} P}{1-\tau_{p}^{k t}}\right\rangle \\
& =\frac{1}{1-\tau_{p}^{k t}}\left\langle\Phi^{k}(P)-\Phi^{k}(\tilde{P}), P-\tilde{P}\right\rangle .
\end{aligned}
$$

Since $\tilde{P}$ also lies on the boundary of the subregion containing $P$, by using the argument in Case (2), we get

$$
\left\langle\Phi^{k}(P)-\Phi^{k}\left(P^{*}\right), P-P^{*}\right\rangle \begin{cases}=0 & \text { if } I_{p}=\emptyset \\ \leq-\frac{1}{\alpha^{k}} \frac{\lambda_{m i n}^{k t}}{2\left(1-\tau_{p}^{k t}\right)}\left\|P_{I_{p}}-P_{I_{p}}^{*}\right\|^{2} & \text { if } I_{p} \neq \emptyset\end{cases}
$$

When $\tau_{p}^{k t} \in(0,1)$ and $I_{i_{k}} \neq \emptyset$, we have

$$
\begin{aligned}
\left\langle\Phi^{k}(P)-\right. & \left.\Phi^{k}\left(P^{*}\right), P-P^{*}\right\rangle=\left\langle\Phi^{k}(P)-\Phi^{k}(\tilde{P})+\Phi^{k}(\tilde{P})-\Phi^{k}\left(P^{*}\right), P-\tilde{P}+\tilde{P}-P^{*}\right\rangle \\
= & \left\langle\Phi^{k}(P)-\Phi^{k}(\tilde{P}), P-\tilde{P}\right\rangle+\left\langle\Phi^{k}(P)-\Phi^{k}(\tilde{P}), \tilde{P}-P^{*}\right\rangle \\
& +\left\langle\Phi^{k}(\tilde{P})-\Phi^{k}\left(P^{*}\right), P-\tilde{P}\right\rangle+\left\langle\Phi^{k}(\tilde{P})-\Phi^{k}\left(P^{*}\right), \tilde{P}-P^{*}\right\rangle . \\
= & \left\langle\Phi^{k}(P)-\Phi^{k}(\tilde{P}), P-\tilde{P}\right\rangle+\frac{\tau_{p}^{k t}}{1-\tau_{p}^{k t}}\left\langle\Phi^{k}(P)-\Phi^{k}(\tilde{P}), P-\tilde{P}\right\rangle \\
& +\frac{1-\tau_{p}^{k t}}{\tau_{p}^{k t}}\left\langle\Phi^{k}(\tilde{P})-\Phi^{k}\left(P^{*}\right), \tilde{P}-P^{*}\right\rangle+\left\langle\Phi^{k}(\tilde{P})-\Phi^{k}\left(P^{*}\right), \tilde{P}-P^{*}\right\rangle . \\
= & \frac{1}{1-\tau_{p}^{k t}}\left\langle\Phi^{k}(P)-\Phi^{k}(\tilde{P}), P-\tilde{P}\right\rangle+\frac{1}{\tau_{p}^{k t}}\left\langle\Phi^{k}(\tilde{P})-\Phi^{k}\left(P^{*}\right), \tilde{P}-P^{*}\right\rangle .
\end{aligned}
$$


By again repeating the argument in Case (2), it is easy to see that $\left\langle\Phi^{k}(P)-\Phi^{k}(\tilde{P}), P-\tilde{P}\right\rangle \leq 0$ and $\left\langle\Phi^{k}(\tilde{P})-\Phi^{k}\left(P^{*}\right), \tilde{P}-P^{*}\right\rangle \leq-\frac{1}{\alpha^{k}} \frac{\lambda_{\text {min }}^{k t}}{2}\left\|\tilde{P}_{I_{i_{k}}}-P_{I_{i_{k}}}^{*}\right\|^{2}$. Substituting these inequalities into (15) and using the relation $\tilde{P}-P^{*}=\tau_{p}^{k t}\left(P-P^{*}\right)$, we obtain

$$
\left\langle\Phi^{k}(P)-\Phi^{k}\left(P^{*}\right), P-P^{*}\right\rangle \leq-\frac{\tau_{p}^{k t} \lambda_{m i n}^{k t}}{2 \alpha_{k}}\left\|P_{I_{i_{k}}}-P_{I_{i_{k}}}^{*}\right\|^{2}
$$

Combining the $\tau_{p}^{k t}=1$ case and the $\tau_{p}^{k t} \in(0,1)$ case, we have

$$
\left\langle\Phi^{k}(P)-\Phi^{k}\left(P^{*}\right), P-P^{*}\right\rangle \begin{cases}\leq 0 & \text { if } I_{i_{k}}=\emptyset \\ \leq-\frac{\tau_{p}^{k t} \lambda_{\min }^{k t}}{2 \alpha_{k}}\left\|P_{I_{i_{k}}}-P_{I_{i_{k}}}^{*}\right\|^{2} & \text { if } I_{i_{k}} \neq \emptyset\end{cases}
$$

The case when $L_{P P^{*}}$ passes through boundaries of one or more subregions between $\Omega_{p}^{k t}$ and $\Omega_{i_{k}}^{k t^{*}}$ can be analyzed similarly by repeatedly applying the above argument and the monotonicity of the mapping $\Phi^{k}$ on each subregion. In particular, let $S_{p}^{k t}$ be the set of intersecting points of $L_{P P^{*}}$ with common boundaries of subregions separating $\Omega_{p}^{k t}$ and $\Omega_{i_{k}}^{k t^{*}}$. Let $\tilde{P}=\arg \min _{P^{\prime} \in S_{p}^{k t}, P^{\prime} \neq P^{*}}\left\|P^{\prime}-P^{*}\right\|$ and $\tau_{p}^{k t}=\frac{\left\|\tilde{P}-P^{*}\right\|}{\left\|P-P^{*}\right\|}$. It can be verified that the same set of inequalities (16) still holds.

Finally, since $\Theta$ is compact by $\mathrm{A} 3, P^{*}$ is fixed, and the number of subregions $\Omega_{p}^{k t}$ is finite, we have $\tau^{k}=\min _{t, p} \tau_{p}^{k t} \geq \frac{\min _{t, p}\left\{\min _{P^{\prime} \in S_{P}^{k t}, P^{\prime} \neq P^{*}}\left\|P^{\prime}-P^{*}\right\|\right\}}{\sup _{P \in \Theta}\left\|P-P^{*}\right\|}>0$ and $\lambda_{\min }^{k}=\min _{t} \lambda_{\min }^{k t}>0$. Thus, Cases (1), (2), and (3) can be combined to yield

$$
\left\langle\Phi^{k}(P)-\Phi^{k}\left(P^{*}\right), P-P^{*}\right\rangle \begin{cases}\leq 0 & \text { if } I_{i_{k}}=\emptyset \\ \leq-\frac{\ell^{k} \lambda_{\min }^{k}}{2 \alpha_{k}}\left\|P_{I_{i_{k}}}-P_{I_{i_{k}}}^{*}\right\|^{2} & \text { if } I_{i_{k}} \neq \emptyset\end{cases}
$$

where $\ell^{k}=1$ in Case (1) and Case (2), and $\ell^{k}=\tau^{k}$ in Case (3).

We now consider the excess demand over all investors. Let $E=\left\{k \mid \Phi^{k}\left(P^{*}\right)=0\right\}$ and $E^{c}=$ $\{1, \cdots, K\} \backslash E$. Clearly, by Assumption A1, $E \neq \emptyset$. Thus by Definition 1, we have from (17) that

$$
\begin{aligned}
\left(\sum_{k=1}^{K} \Phi^{k}(P)-\sum_{k=1}^{K} N^{k}\right)^{\top}\left(P-P^{*}\right) & =\sum_{k=1}^{K}\left(\Phi^{k}(P)-\Phi^{k}\left(P^{*}\right)\right)^{\top}\left(P-P^{*}\right) \\
& \leq \sum_{k \in E^{c}}\left(\Phi^{k}(P)-\Phi^{k}\left(P^{*}\right)\right)^{\top}\left(P-P^{*}\right) \\
& \leq \sum_{k \in E^{c}}-\frac{\ell^{k} \lambda_{m i n}^{k}}{2 \alpha^{k}} \cdot\left\|P_{I_{i_{k}}}-P_{I_{i_{k}}}^{*}\right\|^{2} \\
& \leq h \sum_{k \in E^{c}}\left\|P_{I_{i_{k}}}-P_{I_{i_{k}}}^{*}\right\|^{2},
\end{aligned}
$$


where we have defined $h=-\min _{k} \frac{\ell^{k} \lambda_{\min }^{k}}{2 \alpha^{k}}$. For any asset $j$, the condition $\sum_{k=1}^{K} n_{j}^{k}>0$ in Assumption A1 implies that there exists at least a $k$ such that $j \in I_{i_{k}}$. Thus, it must be the case that $\cup_{k \in E^{c}} I_{i_{k}}=\{1, \cdots, J\}$. Consequently, we obtain $\sum_{k \in E^{c}}\left\|P_{I_{i_{k}}}-P_{I_{i_{k}}}^{*}\right\|^{2} \geq\left\|P-P^{*}\right\|^{2}$. This inequality, when combined with (18), gives the desired result

$$
\left(\sum_{k=1}^{K} \Phi^{k}(P)-\sum_{k=1}^{K} N^{k}\right)^{\top}\left(P-P^{*}\right) \leq h \cdot \sum_{k \in E^{c}}\left\|P_{I_{i_{k}}}-P_{I_{i_{k}}}^{*}\right\|^{2} \leq h \cdot\left\|P-P^{*}\right\|^{2} .
$$

We have the following convergence theorem for the proposed algorithm.

Theorem 1. Let $\left\{a_{n}\right\}$ be a positive sequence satisfying (5). If Assumptions A1-A4 hold and $r$ satisfies $\min _{k=1, \ldots, K}\left(\lambda_{\text {min }}^{k}+r \tilde{\lambda}_{\text {min }}^{k}\right)>0$, then the sequence $\left\{P_{n}\right\}$ generated by Algorithm 1 converges to $P^{*}$ as $n \rightarrow \infty$.

Proof. Let $P_{n+1}$ be the price vector generated at Step 2 of Algorithm 1 at the $n$th iteration. Since $\Pi_{\Theta}\left(P^{*}\right)=P^{*}$ by Assumption A4, we have

$$
\begin{aligned}
& \left\|P_{n+1}-P^{*}\right\|^{2}=\left\|\Pi_{\Theta}\left(P_{n}+a_{n}\left(\sum_{k=1}^{K} \Phi_{n}^{k *}-\sum_{k=1}^{K} N^{k}\right)\right)-\Pi_{\Theta}\left(P^{*}\right)\right\|^{2} \\
& \leq\left\|P_{n}+a_{n}\left(\sum_{k=1}^{K} \Phi_{n}^{k *}-\sum_{k=1}^{K} N^{k}\right)-P^{*}\right\|^{2} \\
& =\left\|P_{n}+a_{n}\left(\sum_{k=1}^{K} \Phi_{n}^{k *}-\sum_{k=1}^{K} \Phi^{k *}\right)-P^{*}\right\|^{2} \text { by Definition } 1 \\
& =\left\|P_{n}-P^{*}\right\|^{2}+2 a_{n}\left(\sum_{k=1}^{K} \Phi_{n}^{k *}-\sum_{k=1}^{K} N^{k}\right)^{\top}\left(P_{n}-P^{*}\right)+\left\|a_{n}\left(\sum_{k=1}^{K} \Phi_{n}^{k *}-\sum_{k=1}^{K} \Phi^{k *}\right)\right\|^{2},
\end{aligned}
$$

where the first inequality above follows from the projection theorem (cf. e.g., Bertsekas and Nedic (2003)). Note that by Lemma 5, the second term in the right-hand-side of (19) is bounded by $\left(\sum_{k=1}^{K} \Phi_{n}^{k *}-\sum_{k=1}^{K} \Phi^{k *}\right)^{\top}\left(P_{n}-P^{*}\right) \leq h\left\|P_{n}-P^{*}\right\|^{2}$ for some constant $h<0$. In addition, by Proposition 1, we have $\left\|\sum_{k=1}^{k} \Phi_{n}^{k *}-\sum_{k=1}^{K} \Phi^{k *}\right\|^{2} \leq \sum_{k=1}^{K}\left\|\Phi_{n}^{k *}-\Phi^{k *}\right\|^{2} \leq \sum_{k=1}^{K} L_{k}\left\|P_{n}-P^{*}\right\|^{2}$. Therefore,

$$
\left\|P_{n+1}-P^{*}\right\|^{2} \leq\left\|P_{n}-P^{*}\right\|^{2}+2 a_{n} h\left\|P_{n}-P^{*}\right\|^{2}+a_{n}^{2} \sum_{k=1}^{K} L_{k}^{2}\left\|P_{n}-P^{*}\right\|^{2}
$$




$$
=\left(1+2 a_{n} h+a_{n}^{2} \sum_{k=1}^{K} L_{k}^{2}\right)\left\|P_{n}-P^{*}\right\|^{2} .
$$

Since $a_{n} \rightarrow 0$ as $n \rightarrow \infty$, there exists $N>0$ such that when $n>N, a_{n}<\frac{-2 h}{\sum_{k=1}^{K} L_{k}^{2}}$, implying $1+2 a_{n} h+a_{n}^{2} \sum_{k=1}^{K} L_{k}^{2}<1$. Thus, by expanding recursion (20), we obtain

$$
\begin{aligned}
\left\|P_{n+1}-P^{*}\right\|^{2} & \leq \prod_{i=1}^{n}\left(1+2 a_{i} h+a_{i}^{2} \sum_{k=1}^{K} L_{k}^{2}\right)\left\|P_{0}-P^{*}\right\|^{2} \\
& =\exp \left(\sum_{i=1}^{n} \ln \left(1+2 a_{i} h+a_{i}^{2} \sum_{k=1}^{K} L_{k}^{2}\right)\right)\left\|P_{0}-P^{*}\right\|^{2} \\
& \leq \exp \left(\sum_{i=1}^{n}\left(2 a_{i} h+a_{i}^{2} \sum_{k=1}^{K} L_{k}^{2}\right)\right)\left\|P_{0}-P^{*}\right\|^{2}
\end{aligned}
$$

where the last inequality above follows from the fact that $\ln (1+x) \leq x \forall x>-1$. Finally, since $h<0, \sum_{i=1}^{\infty} a_{i}=\infty$ and $\sum_{i=1}^{\infty} a_{i}^{2}<\infty$, we have $\lim _{n \rightarrow \infty}\left\|P_{n+1}-P^{*}\right\|=0$, which completes the proof.

\section{$5 \quad$ Numerical Results}

In this section, we begin by testing the robustness and convergence behavior of the algorithm on a set of randomly generated problem instances. We then conduct an empirical sensitivity analysis on a simple 3-investor, 3-asset model to illustrate the application of the proposed algorithm. Next, the performance of the algorithm is compared with that of a popular fixed point method and the well-known branch-and-bound algorithm on problems with different sizes. Finally, we investigate the complexity of the algorithm by reporting the computational time needed to solve relatively large problem instances with up to 100 investors and tradable assets. All computations were performed on a Intel Dual-Core 2.5GHz CPU Windows machine with 2GB of RAM.

\subsection{Convergence Behavior}

We apply the algorithm to a set of 200 randomly generated problem instances, each with 5 investors and 5 risky assets. In our experiments, we set the interest rate $r=0.1$ and use a constant margin requirement of $m_{j}^{k}=0.5$ for all $j$ and $k$. For simplicity, the feasible regions $\Theta$ to all problems are taken to be $[0,1000]^{J}$. In each test problem, the entries of the mean vector $\mu^{k}$ are uniformly selected from the interval $[0.5,1.5]$, and the covariance matrix $\Sigma^{k}$ is constructed by randomly 
generating a $J \times J$ matrix $S^{k}$ with entries uniformly distributed between -1 and 1 and then taking $\Sigma^{k}=S^{k} \times\left(S^{k}\right)^{\top}$. Each random instance generated is then tested for the positive definiteness of $\Sigma^{k}$.

In all test cases, our algorithm is implemented by taking the initial price vector $P_{0}$ to be the average of the expected returns, i.e., $P_{0}=\frac{1}{K} \sum_{k=1}^{K} \mu^{k}$. The gain sequence $\left\{\alpha_{k}\right\}$ is chosen to be of the form $\alpha_{k}=a /(k+A)^{\beta}$ as recommended in Spall (2003). Note that a closer inspection of the performance bound (21) in the proof of Theorem 1 suggests that a slower decay rate in $\alpha_{k}$ could lead to better asymptotic performance. Therefore, we have set $\beta=0.55$ in our experiments. Intuitively, such a slow decay rate ensures that the step size is non-negligible as the number of iteration increases and thus helps to prevent sluggish performance of the algorithm. The constants $a$ and $A$ are chosen together to prevent the initial step sizes from getting too large in the hope to prevent unstable behavior such as oscillation in early iterations of the algorithm. We have experimented with different sets of $a$ and $A$ through some pilot runs of the algorithm on randomly generated problem instances with different sizes. We found empirically that the performance of the algorithm is not sensitive to the choice of $a$, in that values between 0.01 and 1 all seem to work well. In contrast, the choice of $A$ typically relies on the size of the problem. For small-sized problems, values of $A$ on the order of few hundreds seem to work well, whereas for relatively large problems, large values of $A$ appear desirable. Throughout our experiments, we set $a=0.1$ and $A=800$.

Figure 1 shows the performance of the algorithm over 200 randomly generated problem instances. In particular, Figure 1(a) plots the differences between demand and supply (i.e., $\| \sum_{k=1}^{K} \Phi_{n}^{k *}-$ $\sum_{k=1}^{K} N^{k} \|$ averaged over 200 random test instances) as a function of the number of algorithm iterations, whereas Figure 1(b) shows the deviations of the solution $P_{n}$ found at the $n$th iteration from the optimal price vector $P^{*}$ as the number of iterations $n$ increases. In both cases, the $99 \%$ confidence bound curves are shown in blue. Note that since the true optimal equilibrium price $P^{*}$ cannot be obtained analytically, in evaluating the deviation $\left\|P_{n}-P^{*}\right\|$ in Figure 1(b), we have approximated $P^{*}$ by $\hat{P}^{*}$, which was computed by running the algorithm for a large number of iterations until the difference $\left\|\sum_{k=1}^{K} \Phi_{n}^{k *}-\sum_{k=1}^{K} N^{k}\right\|$ falls below $10^{-4}$. From the small differences between demand and supply at the end of iterations, we see that the algorithm consistently converges to the respective equilibrium solutions to the 200 randomly generated instances. In particular, the figure shows that the algorithm converges rapidly to a small neighborhood of an optimal solution within the first few tens of iterations and then spends most of the effort in fine-tuning the solution. In addition, as indicated by the gradually decreasing width of confidence intervals, the variability 


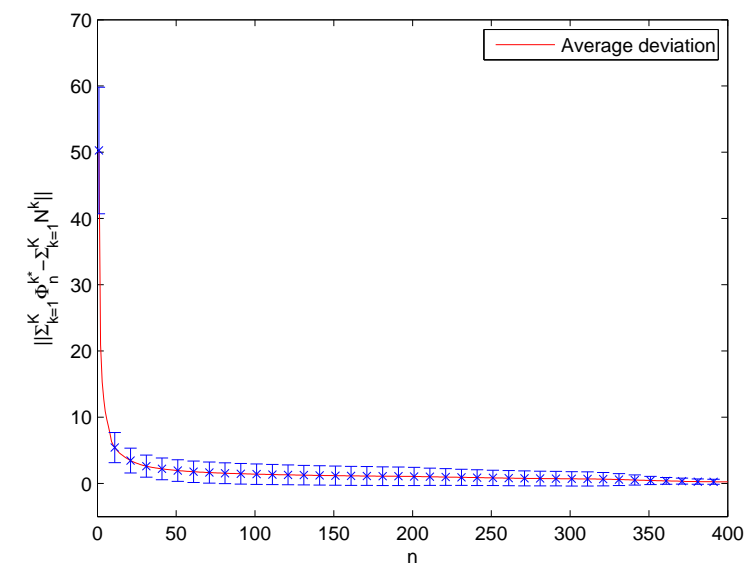

(a)

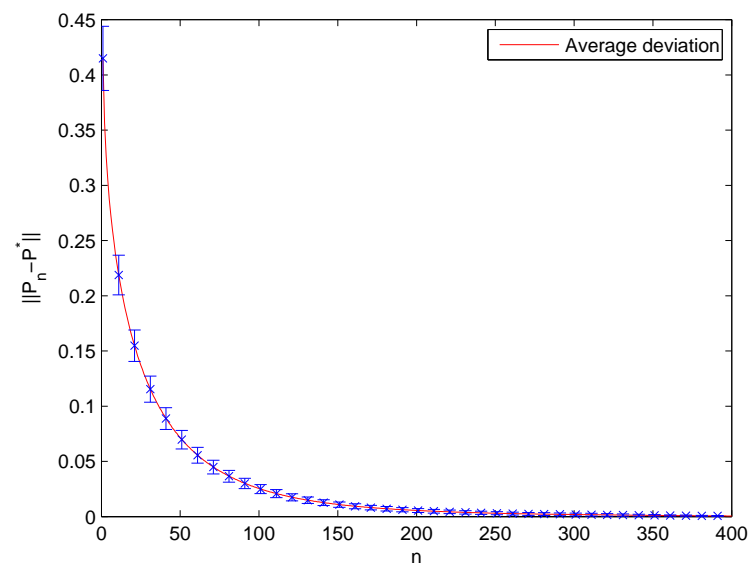

(b)

Figure 1: Average performance of the algorithm on 200 randomly generated test instances with 5 investors and 5 assets. 99\% confidence bounds are plotted every 10 iterations in blue.

in algorithm performance vanishes as the number of iterations increases.

\subsection{Sensitivity Analysis}

To exemplify the potential application of the proposed algorithm, we apply it to a simple example to investigate the impact of marginal requirements and interest rates on the resulting equilibrium prices. The market under consideration consists of three investors and three assets with $\alpha^{1}=\alpha^{2}=$ $\alpha^{3}=1$. The initial endowments and individual beliefs are given respectively by $N^{1}=(1,0,0)^{\top}$, $N^{2}=(0,1,0)^{\top}, N^{3}=(0,0,1)^{\top}, E^{1}[X]=(3,3,1)^{\top}, E^{2}[X]=(1,2,1)^{\top}, E^{3}[X]=(1,2,3)^{\top}$, and

$$
\Sigma^{1}=\left(\begin{array}{lll}
1 & 1 & 1 \\
1 & 2 & 1 \\
1 & 1 & 3
\end{array}\right), \Sigma^{2}=\left(\begin{array}{lll}
3 & 1 & 1 \\
1 & 1 & 1 \\
1 & 1 & 2
\end{array}\right), \Sigma^{3}=\left(\begin{array}{ccc}
3 & 1 & 1 \\
1 & 2 & 1 \\
1 & 1 & 1
\end{array}\right)
$$

Tests were performed on the model by varying the values of the margin requirement $m$ and interest rate $r$. In each case, we stop the algorithm when the difference $\left\|\sum_{k=1}^{K} \Phi_{n}^{k *}-\sum_{k=1}^{K} N^{k}\right\|$ falls below 0.01. Figure 2(a) shows the equilibrium prices obtained when using various values of $m$ (from 0.05 to 1 , with an increment of 0.05 ) and $r$ (from 0.01 to 0.2 , with an increment of 0.01 ). From the figure, we see that the prices of all assets are monotone with respect to both $m$ and $r$, indicating that both the margin requirement and interest rate should have an impact on the equilibrium prices. In particular, Figure 2(b) shows that when the interest rate is fixed at 0.05 , the price of asset 1 

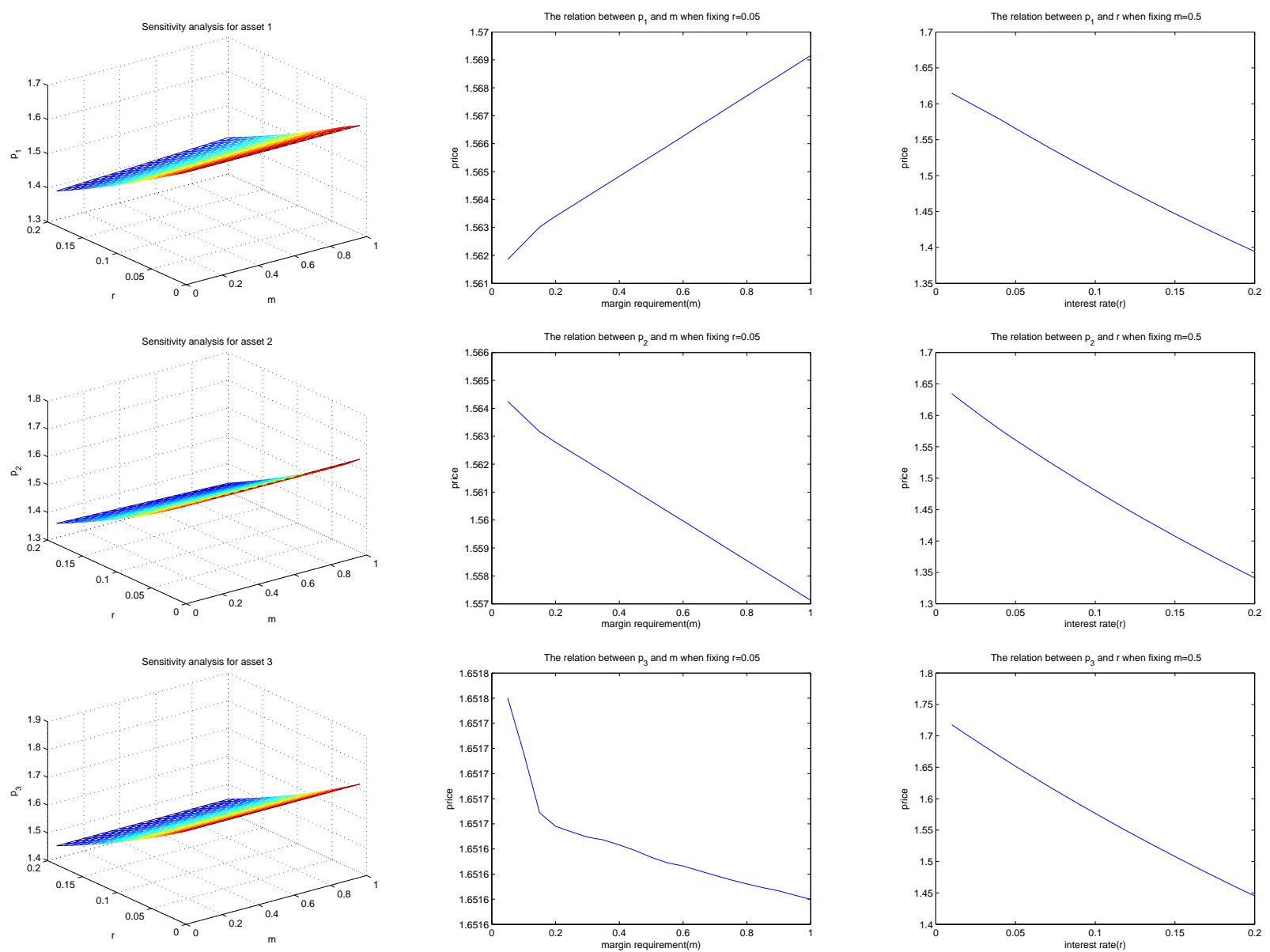

(a)

(b)

(c)

Figure 2: (a) joint effect of interest rates and margin requirements on equilibrium prices; (b) marginal effect of margin requirements on equilibrium prices when the interest rate is fixed at 0.05 ; (c) marginal effect of interest rates on equilibrium prices when the marginal requirement is fixed at 0.5 .

increases monotonically with $m$, while the prices of assets 2 and 3 decrease monotonically. On the other hand, as indicated in Figure 2(c), for a fixed margin requirement $m=0.5$, the prices of all assets decrease as the interest rate increases. In addition, notice that the relative rates of change of prices with respect to $m$ are much slower than those with respect to $r$. This suggests that compared to the interest rate, margin requirements may have less influence on the resulting equilibrium prices. Although no general conclusion can be drawn in this particular case, this example serves to illustrate that the proposed algorithm may be used as a simple, straightforward tool to investigate specific market models. 


\subsection{Comparison with A Fixed Point Algorithm}

We compare our algorithm with the variable dimension restart algorithm of Laan and Talman (1979), which is considered as one of the most efficient algorithms within the fixed point framework. The main idea behind the algorithm is to subdivide the entire domain into a number of $m$ sub-simplices. Starting from an initial point, the algorithm generates a path of adjacent $t$-simplices of varying dimension and terminates with a completely labeled simplex that is guaranteed to contain the true fixed point. We use the arithmetic mean of the completely labeled vertexes as an approximation of the true equilibrium price vector. Because its distance to the true fixed point is upper bounded by the mesh size, the accuracy of the approximation can be improved by increasing the grid size $m$.

To cast our problem into a fixed point framework, we note that finding an equilibrium price vector of our model is equivalent to finding a fixed point $P^{*}$ to the equation $f\left(P^{*}\right)=P^{*}$, where

$$
f(P)=\sum_{k=1}^{K} \Phi^{k}(P)-\sum_{k=1}^{K} N^{k}+P .
$$

By Lemma $2, f(P)$ is a continuous mapping from $\Re^{J}$ to $\Re^{J}$, which satisfies the existence condition of fixed point theorem. The following integer labeling rule is used when implementing the algorithm of Laan and Talman (1979):

$$
l(P)=\min \left\{i \mid p_{i}>0, f_{i}(P)-p_{i} \leq f_{k}(P)-p_{k}, \forall k \in\{1, \cdots, J\}\right\} .
$$

The following two examples are used in our comparison study: (i) a two-investor, two-asset problem with parameters $\alpha^{1}=\alpha^{2}=1$, where the initial endowments and the beliefs for individuals are, respectively, $N^{1}=(1,0), N^{2}=(0,1), E^{1}[X]=(2,1), E^{2}[X]=(1,3)$, and

$$
\Sigma^{1}=\left(\begin{array}{ll}
1 & 1 \\
1 & 3
\end{array}\right), \Sigma^{2}=\left(\begin{array}{ll}
3 & 1 \\
1 & 1
\end{array}\right)
$$

(ii) a three-investor, three-asset problem, where the risk aversion parameters, initial endowments, and individual beliefs are taken to be the same as those in Section 5.2. In both cases, we set the interest rate $r=0.1$ and use a constant margin requirement of $m_{j}^{k}=0.5$ for all $j$ and $k$.

Table 1 shows the numbers of iterations and the running time required for the fixed point algorithm to find an approximated equilibrium price vector when using different mesh sizes for 
Table 1: Performance of the fixed point algorithm on test problems $(i)$ and $(i i)$ with initial points $(0.2,0.8)$ in case $(i)$ and $(0.2,0.3,0.5)$ in case $(i i)$.

\begin{tabular}{|c|c|c|c|c|c|c|}
\hline Cases & $m$ & Mesh size & Error & \#iterations & Time(s) & Equilibrium prices \\
\hline \multirow{3}{*}{$(i)$} & 10 & 0.1414 & 0.1170 & 26 & 36.8 & $(1.1250,1.7500)$ \\
& $10^{2}$ & 0.0141 & 0.0096 & 222 & 277.6 & $(1.0525,1.6850)$ \\
& $10^{3}$ & 0.0014 & $5.32 \times 10^{-4}$ & 2186 & $2.75 \times 10^{3}$ & $(1.0465,1.6813)$ \\
& $10^{4}$ & $1.4142 \times 10^{-4}$ & $\mathrm{~N} / \mathrm{A}$ & $\mathrm{N} / \mathrm{A}$ & $>12 h$ & $\mathrm{~N} / \mathrm{A}$ \\
\hline \multirow{3}{*}{ (ii $)$} & 10 & 0.1732 & 0.1342 & 54 & 111.7 & $(1.5600,1.5400,1.6200)$ \\
& $10^{2}$ & 0.0173 & 0.0315 & 506 & $1.08 \times 10^{3}$ & $(1.5140,1.4920,1.5860)$ \\
& $10^{3}$ & 0.0017 & 0.0021 & 4977 & $1.11 \times 10^{4}$ & $(1.4996,1.4764,1.5722)$ \\
\hline
\end{tabular}

Table 2: Performance of the proposed algorithm with initial points $(0.2,0.8)$ in case $(i)$ and $(0.2,0.3,0.5)$ in case $(i i)$.

\begin{tabular}{|c|c|c|c|c|c|}
\hline Cases & $\epsilon$ & Error & \#iterations & Time(s) & Equilibrium prices \\
\hline \multirow{3}{*}{$(i)$} & 0.1170 & 0.1135 & 3 & 2.1 & $(1.0697,1.7068)$ \\
& 0.0096 & 0.0090 & 12 & 8.3 & $(1.0514,1.6864)$ \\
& $5.32 \times 10^{-4}$ & $5.31 \times 10^{-4}$ & 145 & 102.4 & $(1.0465,1.6814)$ \\
& $10^{-4}$ & $9.99 \times 10^{-5}$ & 659 & 10212.7 & $(1.0462,1.6812)$ \\
\hline \multirow{3}{*}{$(i i)$} & 0.1342 & 0.1135 & 7 & 8.7 & $(1.5160,1.4970,1.5950)$ \\
& 0.0315 & 0.0304 & 10 & 11.9 & $(1.5126,1.4893,1.5857)$ \\
& 0.0021 & 0.0021 & 103 & 117.0 & $(1.4998,1.4764,1.5725)$ \\
\hline
\end{tabular}

each of the respective test problems $(i)$ and $(i i)$, where the approximation error is measured by the difference $\left\|\sum_{k=1}^{K} \Phi_{n}^{k *}-\sum_{k=1}^{K} N^{k}\right\|$. We see from the table that the approximation error reduces as the number of grids increases. However, both the number of iterations and the computational time increase drastically each time the mesh size is reduced to one-tenth of its previous value. In particular in case $(i)$, when the grid size reaches $10^{4}$, the algorithm runs for more than 12 hours without returning a solution.

Table 2 gives the performance of the proposed algorithm on both test problems using the gain sequence $a_{n}=1 / n$. In each of the respective test cases, we stop the algorithm when the difference $\left\|\sum_{k=1}^{K} \Phi_{n}^{k *}-\sum_{k=1}^{K} N^{k}\right\|$ falls below a given tolerance $\epsilon$, where $\epsilon$ is set to the approximation error value shown in the corresponding entry of Table 1. In other words, we stop the algorithm when it reaches an approximation error that is no greater than that obtained by the fixed point algorithm. From the tables, we see that the reduction in computational time of our algorithm over the fixed point algorithm is a factor of between 10 and 100, providing many-fold savings in computational cost. 


\subsection{Comparison with Branch-and-Bound}

We also compare our algorithm with the branch-and-bound algorithm, which is popular in handling problems with complementarity constraints. To apply the branch-and-bound algorithm, we first cast our model into the following equivalent form:

$$
\begin{aligned}
& \min _{P,\left\{\Phi^{k}, \Psi^{k}, \lambda^{k}, \eta^{k}\right\}_{k=1}^{K}}\left\|\sum_{k=1}^{K} \Phi^{k}-\sum_{k=1}^{K} N^{k}\right\| \\
& \text { subject to } \alpha^{k} \Sigma^{k} \Phi^{k}-\left(\mu^{k}-\Lambda^{k} P\right)+\lambda^{k}=0, \\
& r \Gamma^{k} P-\left(\lambda^{k}+\eta^{k}\right)=0, \\
& \lambda_{i}^{k}\left(\phi_{i}^{k}-\psi_{i}^{k}\right)=0, i=1, \cdots, J ; k=1, \cdots, K \\
& \eta_{i}^{k} \psi_{i}^{k}=0, i=1, \cdots, J ; k=1, \cdots, K \\
& \Psi^{k} \geq \Phi^{k}, \\
& P \geq 0, \Phi^{k} \in \Re^{J}, \Psi^{k} \geq 0, \lambda^{k} \geq 0, \eta^{k} \geq 0, k=1, \cdots, K .
\end{aligned}
$$

The constraints above are the KKT conditions for the optimal portfolio selection problem (9) of each investor for all $k$, where $\lambda^{k}$ and $\eta^{k}$ are dual variables. It is easy to see that any feasible solution of this problem must also solve the portfolio selection problem (9) for all $k$. The objective function measures the gap between market demand and supply, which captures the equilibrium prices when it attains the minimum value zero.

Because problems with complementary slackness constraints are often nonlinear, standard nonlinear programming algorithms in general can only obtain local optimal solutions. So a commonly used approach is to transform the problem into an equivalent mixed integer programming problem (cf. e.g., Hu et al. (2012)), which in our case can be written as:

$$
\begin{gathered}
\min _{P,\left\{\Phi^{k}, \Psi^{k}, \lambda^{k}, \eta^{k}\right\}_{k=1}^{K},\left\{z_{i}^{k}, q_{i}^{k}\right\}_{i=1, k=1}^{J, K}}\left\|\sum_{k=1}^{K} \Phi^{k}-\sum_{k=1}^{K} N^{k}\right\| \\
\text { subject to } \alpha^{k} \Sigma^{k} \Phi^{k}-\left(\mu^{k}-\Lambda^{k} P\right)+\lambda^{k}=0 \\
r \Gamma^{k} P-\left(\lambda^{k}+\eta^{k}\right)=0 \\
0 \leq \lambda_{i}^{k} \leq \bar{\lambda}_{i}^{k} z_{i}^{k} \\
\left(1-z_{i}^{k}\right) d_{i}^{k} \leq \phi_{i}^{k}-\psi_{i}^{k} \leq 0 \\
0 \leq \eta_{i}^{k} \leq \bar{\eta}_{i}^{k} q_{i}^{k}
\end{gathered}
$$




$$
\begin{aligned}
& 0 \leq \psi_{i}^{k} \leq\left(1-q_{i}^{k}\right) \bar{\psi}_{i}^{k}, \\
& z_{i}^{k}, q_{i}^{k} \in\{0,1\}, i=1, \cdots, J ; k=1, \cdots, K . \\
& P \geq 0, \Phi^{k} \in \Re^{J}, k=1, \cdots, K
\end{aligned}
$$

where $\bar{\lambda}_{i}^{k}$ and $\bar{\eta}_{i}^{k}$ represent the upper bounds on variables $\lambda_{i}^{k}$ and $\eta_{i}^{k}$, and $d_{i}^{k}$ denotes a lower bound on $\phi_{i}^{k}-\psi_{i}^{k}$ for all $i$ and $k$, all of which need to be estimated and used as inputs to the model; cf., e.g., Hu et al. (2012). The problem can then be solved by using the standard branch-and-bound algorithm.

\begin{tabular}{|c|c|c|c|c|c|c|}
\hline & \multicolumn{3}{|c|}{ Our Algorithm } & \multicolumn{3}{c|}{ Branch-and-Bound } \\
\hline$(\mathrm{K}, \mathrm{J})$ & \#Iterations & Time(s) & Error & \#Nodes & Time(s) & Error \\
\hline$(5,5)$ & 116 & 234.6 & 0.0098 & 1229 & 1.1 & $4.42 \times 10^{-14}$ \\
$(8,8)$ & 201 & 777.7 & 0.0097 & 1531 & 5.9 & $1.58 \times 10^{-13}$ \\
$(10,10)$ & 161 & 701.5 & 0.0099 & $>34859$ & $>1800$ & 591.31 \\
$(15,15)$ & 318 & 1792.8 & 0.0096 & N/A & N/A & N/A \\
\hline
\end{tabular}

Table 3: Comparison with the branch-and-bound algorithm

In our numerical comparison, the lower bound $d_{i}^{k}$ is set to -10000 and the upper bounds $\bar{\lambda}_{i}^{k}$ and $\bar{\eta}_{i}^{k}$ are set to 10000 for all $i$ and $k$, and the branch-and-bound algorithm is implemented using the commercial optimization package CPLEX. Table 3 shows the results obtained by our proposed algorithm and branch-and-bound on four randomly generated problem instances. In each case, we stop our algorithm when the error $\left\|\sum_{k=1}^{K} \Phi_{n}^{k *}-\sum_{k=1}^{K} N^{k}\right\|$ falls below 0.01, whereas the branch-andbound algorithm is stopped whenever the gap between the best integer objective and the objective of the best node remaining in the branch-and-bound tree falls below 0.01. Empirical results indicate superior performance of branch-and-bound when the problem is small. In particular, in the first two test cases, the algorithm finds highly accurate solutions within a few seconds. However, since the number of nodes in the branch-and-bound tree grows exponentially with the numbers of investors and assets, the algorithm quickly becomes computationally inefficient as the problem size increases. We see that in the case when $K=10$ and $J=10$, the algorithm runs for more than 1800 seconds and fails to return an acceptable solution after searching 34859 nodes.

\subsection{Computational Complexity}

To investigate the computational complexity of the algorithm, tests were performed on CAPM models with increasing numbers of investors and tradable assets; for each model of a given size, 30 problem instances are randomly generated. Table 4 reports the minimum, maximum, and averaged 
computational time (over 30 random instances) required for the algorithm to find an approximate equilibrium price for each of the respective cases, where in all runs, the algorithm is terminated when the difference $\left\|\sum_{k=1}^{K} \Phi_{n}^{k *}-\sum_{k=1}^{K} N^{k}\right\|$ is smaller than 0.01. We see from the table that the algorithm scales well with the problem size and can solve relatively large problem instances within a moderate amount of time with little compromise on the optimality of the solution. We remark that the computational time of the algorithm is primarily dominated by the time required to carry out Step 1, where an optimal portfolio selection problem needs to be solved $K$ times. So the performance of the algorithm can be significantly improved by using a parallel implementation scheme to solve the $K$ portfolio selection problems simultaneously.

Table 4: Computational time (in seconds) of the algorithm on models with increasing numbers of investors and assets. Each case is based on 30 randomly generated instances.

\begin{tabular}{|c|c|c|c|c|c|}
\hline Cases & \#investors & \# assets & Min. time & Max. time & Avg. time \\
\hline 1 & 5 & 5 & 226.7 & 971.1 & 532.1 \\
2 & 10 & 10 & 448.5 & $1.36 \times 10^{3}$ & 806.8 \\
3 & 20 & 20 & 774.5 & $1.49 \times 10^{3}$ & $1.14 \times 10^{3}$ \\
4 & 50 & 50 & $1.72 \times 10^{3}$ & $2.29 \times 10^{3}$ & $1.98 \times 10^{3}$ \\
5 & 100 & 100 & $3.72 \times 10^{3}$ & $4.49 \times 10^{3}$ & $3.96 \times 10^{3}$ \\
\hline
\end{tabular}

\section{Conclusions}

In this paper, we have studied a capital asset pricing model with heterogeneous investors and arbitrary margin requirements. Unlike existing work in the area, which has concentrated on deriving analytical solutions to the model under simplifying assumptions, we have proposed a general derivative-free computational algorithm for iteratively estimating the optimal equilibrium price vector. We have analyzed the structural property of the optimal portfolio selection function and shown that it is Lipschitz continuous and piece-wise linear in the underlying price vector. In addition, under some appropriate conditions, we have also established the monotonicity of the market excess demand function around the optimal price vector. These properties allow us to show the convergence of the algorithm by explicitly bounding the distance between an incumbent solution found at the $n$th iteration and the optimal equilibrium price vector. Numerical results indicate that the algorithm may yield superior performance over the fixed point method and the popular branch-and-bound algorithm, and is capable of producing high-quality solutions very close to the optimum within moderate computational time. 


\section{Acknowledgments}

This work was supported by the National Natural Science Foundation of China (NSFC) under

Grants 71571048, 71071040, by the Science and Technology Agency of Sichuan Province under Grant 2014GZX0002, by the Program for Professor of Special Appointment (Eastern Scholar) at Shanghai Institution of Higher Learning. The work of Jiaqiao Hu was supported by the National Science Foundation under Grant CMMI-1130761.

\section{References}

Anufriev, M., J. Tuinstra. 2013. The impact of short-selling constraints on financial market stability in a heterogeneous agents model. Journal of Economic Dynamics \& Control 37 1523-1543.

Arrow, K.J., L. Hurwicz. 1960. Some remarks on the equilibria of economic systems. Econometrica 28 640-646.

Bertsekas, D., A. Nedic. 2003. Convex analysis and optimization. Athena Scientific.

Chiarella, C., R. Dieci, X.-Z. He, K. Li. 2013. An evolutionary capm under heterogeneous beliefs. Annals of Finance 9 185-215.

Cottle, R.W., J.-S. Pang, R.E. Stone. 1992. The linear complementarity problem. SIAM.

Dafermos, S. 1990. Exchange price equilibria and variational inequalities. Mathematical Programming 46 391-402.

Eaves, B.C. 1972. Homotopies for computation of fixed points. Mathematical Programming 3 1-22.

Eaves, B.C., K. Schmedders. 1999. General equilibrium models and homotopy methods. Journal of Economic Dynamics \& Control 23 1249-1279.

Fama, E.F., K.R. French. 2007. Disagreement, tastes, and asset prices. Journal of Financial Economics 83 667-689.

Garg, R., S. Kapoor. 2006. Auction algorithms for market equilibrium. Mathematics of Operations Research 31 714-729.

Gârleanu, N., L. H. Pedersen. 2011. Margin-based asset pricing and deviations from the law of one price. Review of Financial Studies 24 1980-2022. 
Ghiyasvand, M., J.B. Orlin. 2012. A simple approximation algorithm for computing arrow-debreu prices. Operations Research 60 1245-1248.

He, X., L. Shi. 2007. Zero-beta capm with heterogeneous beliefs. 20th Australasian Finance \& Banking Conference .

Horn, R.A., C.R. Johnson. 2012. Matrix analysis. Cambridge University Press.

Hu, J., J.E. Mitchell, J.-S. Pang, B. Yu. 2012. On linear programs with linear complementarity constraints. Journal of Global optimization $5329-51$.

Jarrow, R. 1980. Heterogeneous expectations, restrictions on short sales, and equilibrium asset prices. The Journal of Finance 35 1105-1113.

Jofré, A., R. T. Rockafellar, R.J-B. Wets. 2007. Variational inequalities and economic equilibrium. Mathematics of Operations Research 32 32-50.

Laan, G. v. d., A.J.J. Talman. 1979. A restart algorithm without an artificial level for computing fixed points on unbounded regions. H.-O. Peitgen, H.-O. Walther, eds., Functional differential equations and approximation of fixed points, vol. 730. Springer Berlin Heidelberg, 247-256.

Lam, K., P.L.H. Yu, P.H. Lee. 2010. A margin scheme that advises on when to change required margin. European Journal of Operational Research 207 524-530.

Levy, H. 1978. Equilibrium in an imperfect market: a constraint on the number of securities in the portfolio. The American Economic Review 68 643-658.

Levy, H., M. Levy, G. Benita. 2006. Capital asset prices with heterogeneous beliefs. The Journal of Business 79 1317-1353.

Levy, M. 2007. Conditions for a capm equilibrium with positive prices. Journal of Economic Theory $137404-415$.

Lintner, J. 1969. The aggregation of investor's diverse judgments and preferences in purely competitive security markets. The Journal of Financial and Quantitative Analysis 4 347-400.

Luo, Z.-Q., J.-S. Pang, D. Ralph. 1996. Mathematical programs with equilibrium constraints. Cambridge University Press. 
Ma, C., J. Hu, Y. Xu. 2015. Short selling and endogenous price uncertainty. Journal of Mathematical Economics under review.

Markowitz, H. 1952. Portfolio selection. The Journal of Finance 7 77-91.

Nielsen, L. T. 1992. Positive prices in capm. The Journal of Finance 47 791-808.

Rytchkov, O. 2014. Asset pricing with dynamic margin constraints. The Journal of Finance 69 405-452.

Scarf, H.E., T. Hansen. 1973. The computation of economic equilibria. Yale University Press.

Shi, L. 2016. Consumption-based capm with belief heterogeneity. Journal of Economic Dynamics \& Control 65 30-46.

Spall, J.C. 2003. Introduction to stochastic search and optimization. John Wiley \& Sons.

Sun, N., Z. Yang. 2003. Existence of equilibrium and zero-beta pricing formula in the capital asset pricing model with heterogeneous beliefs. Annals of Economics and Finance 4 51-71.

Walras, L. 1954. Elements of pure economics: or the theory of social wealth. Taylor \& Francis. English translation by W.Jaffé.

Yen, N.D. 1995. Lipschitz continuity of solutions of variational inequalities with a parametric polyhedral constraint. Mathematics of Operations Research 20 695-708. 